Rakesh Arora, Jacques Giacomoni*, and Guillaume Warnault

\title{
A Picone identity for variable exponent operators and applications
}

https://doi.org/10.1515/anona-2020-0003

Received October 27, 2018; accepted November 29, 2018.

Abstract: In this work, we establish a new Picone identity for anisotropic quasilinear operators, such as the $p(x)$-Laplacian defined as $\operatorname{div}\left(|\nabla u|^{p(x)-2} \nabla u\right)$. Our extension provides a new version of the Diaz-Saa inequality and new uniqueness results to some quasilinear elliptic equations with variable exponents. This new Picone identity can be also used to prove some accretivity property to a class of fast diffusion equations involving variable exponents. Using this, we prove for this class of parabolic equations a new weak comparison principle.

Keywords: parabolic problems with non standard growth, Picone identity for varible exponents, uniqueness, global behaviour

MSC: 35A16, 35B51, 35K92, 35J92

\section{Introduction and main results}

The main aim of this paper is to prove a new version of the Picone identity involving quasilinear elliptic operators with variable exponent. The Picone identity is already known for homogeneous quasilinear elliptic as $p$-Laplacian with $1<p<\infty$.

In [1], M. Picone considers the homogeneous second order linear differential system

$$
\left\{\begin{array}{l}
\left(a_{1}(x) u^{\prime}\right)^{\prime}+a_{2}(x) u=0 \\
\left(b_{1}(x) v^{\prime}\right)^{\prime}+b_{2}(x) v=0
\end{array}\right.
$$

and proved for differentiable functions $u, v \neq 0$ the pointwise relation:

$$
\left(\frac{u}{v}\left(a_{1} u^{\prime} v-b_{1} u v^{\prime}\right)\right)^{\prime}=\left(b_{2}-a_{2}\right) u^{2}+\left(a_{1}-b_{1}\right) u^{\prime 2}+b_{1}\left(u^{\prime}-\frac{v^{\prime} u}{v}\right)^{2}
$$

and in [2], extended (1.1) to the Laplace operator, i.e. for differentiable functions $u \geq 0, v>0$ one has

$$
|\nabla u|^{2}+\frac{u^{2}}{v^{2}}|\nabla v|^{2}-2 \frac{u}{v} \nabla u . \nabla v=|\nabla u|^{2}-\nabla\left(\frac{u^{2}}{v}\right) \cdot \nabla v \geq 0 .
$$

In [3], Allegretto and Huang extended (1.2) to the $p$-Laplacian operator with $1<p<\infty$. Precisely, for differentiable functions $v>0$ and $u \geq 0$ we have

$$
|\nabla u|^{p}-|\nabla v|^{p-2} \nabla v \cdot \nabla\left(\frac{u^{p}}{v^{p-1}}\right) \geq 0
$$

Rakesh Arora, LMAP (UMR E2S-UPPA CNRS 5142) Bat. IPRA, Avenue de l’Université 64013 Pau, France,

E-mail: rakesh.arora@univ-pau.fr

*Corresponding Author: Jacques Giacomoni, LMAP (UMR E2S-UPPA CNRS 5142) Bat. IPRA, Avenue de l'Université 64013 Pau,

France, E-mail: jacques.giacomoni@univ-pau.fr

Guillaume Warnault, LMAP (UMR E2S-UPPA CNRS 5142) Bat. IPRA, Avenue de l’Université 64013 Pau, France,

E-mail: guillaume.warnault@univ-pau.fr 
Picone identity plays an important role for proving qualitative properties of differential operators. In this regard, various attempts have been made to generalize Picone identity for different types of differential equations. At the same time, the study of differential equations and variational problems with variable exponents are getting more and more attention. Indeed, the mathematical problems related to nonstandard $p(x)$-growth conditions are connected to many different areas as the nonlinear elasticity theory and non-Newtonian fluids models (see [4, 5]). In particular the importance of investigating these kinds of problems lies in modelling various anisotropic features that occur in electrorheological fluids models, image restoration [6], filtration process in complex media, stratigraphy problems [7] and heterogeneous biological interactions [8]. The mathematical framework to deal with these problems are the generalized Orlicz Space $L^{p(x)}(\Omega)$ and the generalized Orlicz-Sobolev Space $W^{1, p(x)}(\Omega)$. We refer to [9-14] for the existence and regularity of minimizers in variational problems.

In $[15,16]$, several applications of Picone-type identity for $p(\cdot)=$ constant case have been obtained. This original identity is not further applicable for differential equations with $p(x)$-growth conditions. So, it is relevant to establish a new version of the Picone identity to include a large class of nonstandard $p(x)$-growth problems. In $[5,12,17]$ convexity arguments to homogeneous functionals have been used to deal with quasilinear elliptic and parabolic problems with variable exponents. In the present paper, taking advantage of our new Picone pointwise identity, we give further applications in the context of elliptic and parabolic problems.

Before giving the statement of our main results, we first introduce notations and function spaces. Let $\Omega \subset \mathbb{R}^{N}, N \geq 1$. We recall some definitions of variable exponent Lebesgue and Sobolev spaces. Let $\mathcal{P}(\Omega)$ be the set of all measurable function $p: \Omega \rightarrow[1, \infty[$ in $N$-dimensional Lebesgue measure. Define

$$
\begin{gathered}
\rho_{p}(u) \stackrel{\text { def }}{=} \int_{\Omega}|u|^{p(x)} d x . \\
L^{p(x)}(\Omega)=\left\{u: \Omega \rightarrow \mathbb{R} \mid u \text { is measurable and } \rho_{p}(u)<\infty\right\}
\end{gathered}
$$

endowed with the norm

$$
\|u\|_{L^{p(x)}}=\inf \left\{\sigma>0: \rho_{p}\left(\frac{u}{\sigma}\right) \leq 1\right\} .
$$

The corresponding Sobolev space is defined as follows:

$$
W^{1, p(x)}(\Omega)=\left\{u \in L^{p(x)}(\Omega):|\nabla u| \in L^{p(x)}(\Omega)\right\}
$$

endowed with the norm

$$
\|u\|_{W^{1, p(x)}}=\|u\|_{L^{p(x)}}+\|\nabla u\|_{L^{p(x)}}
$$

and $W_{0}^{1, p(x)}(\Omega)=W_{0}^{1,1}(\Omega) \cap W^{1, p(x)}(\Omega)$.

In the sequel, we assume that $\Omega$ satisfies:

( $\Omega$ ) For $N=1, \Omega$ is a bounded open interval and for $N \geq 2, \Omega$ is a bounded domain whose the boundary $\partial \Omega$ is a compact manifold of class $C^{1, y}$ for some $y \in(0,1)$ and satisfies the interior sphere condition at every point of $\partial \Omega$.

Throughout the paper, we also assume that $p \in C^{1}(\bar{\Omega})$. In addition, we suppose that

$$
1<p_{-} \stackrel{\text { def }}{=} \min _{\bar{\Omega}} p(x) \leq p(x) \leq p_{+} \stackrel{\text { def }}{=} \max _{\bar{\Omega}} p(x)<\infty .
$$

Then, $W_{0}^{1, p(x)}(\Omega)={\overline{C_{0}^{\infty}(\Omega)}}^{W^{1, p(x)}(\Omega)}$. We also recall some well-known properties on $L^{p(x)}$ spaces (see [14]).

Proposition 1.1. Let $p \in L^{\infty}(\Omega)$. Then for any $u \in L^{p(x)}(\Omega)$ we have:

(i) $\quad \rho_{p}\left(u /\|u\|_{L^{p(x)}}\right)=1$.

(ii) $\|u\|_{L^{p(x)}} \rightarrow 0$ if and only if $\rho_{p}(u) \rightarrow 0$. 


$$
p_{c}(x)=\frac{p(x)}{p(x)-1}
$$

Proposition 1.1 (i) implies that: if $\|u\|_{L^{p(x)}} \geq 1$,

$$
\|u\|_{L^{p(x)}}^{p_{-}} \leq \rho_{p}(u) \leq\|u\|_{L^{p(x)}}^{p_{+}}
$$

and if $\|u\|_{L^{p(x)}} \leq 1$

$$
\|u\|_{L^{p(x)}}^{p_{+}} \leq \rho_{p}(u) \leq\|u\|_{L^{p(x)}}^{p_{-}} .
$$

Moreover, we have also the generalized Hölder inequality: for $p$ measurable function in $\Omega$, there exists a constant $C=C\left(p_{+}, p_{-}\right) \geq 1$ such that for any $f \in L^{p(x)}(\Omega)$ and $g \in L^{p_{c}(x)}(\Omega)$

$$
\int_{\Omega}|f(x) g(x)| d x \leq C\|f\|_{L^{p(x)}}\|g\|_{L^{p_{c}(x)}} \text {. }
$$

In Section 2, we prove the Picone identity for a general class of nonlinear operator. More precisely, we consider a continuous operator $A: \Omega \times \mathbb{R}^{N} \rightarrow \mathbb{R}$ such that $(x, \xi) \rightarrow A(x, \xi)$ is differentiable with respect to variable $\xi$ and satisfies:

$$
\begin{aligned}
& \xi \rightarrow A(x, \xi) \text { is positively } p(x) \text {-homogeneous i.e. } A(x, t \xi)=t^{p(x)} A(x, \xi), \forall t \\
& \in \mathbb{R}^{+}, \xi \in \mathbb{R}^{N} \text { and a.e. } x \in \Omega . \\
& \xi \rightarrow A(x, \xi) \text { is strictly convex for any } x \in \Omega .
\end{aligned}
$$

Remark 1.1. From the assumptions of $A$, we deduce $A(x, \xi)>0$ for $\xi \neq 0$ and for any $x \in \Omega$.

By using the convexity and the $p(x)$-homogeneity of the operator $A$, we prove the following extension of the Picone identity:

Theorem 1.1 (Picone identity). Let $A: \Omega \times \mathbb{R}^{N} \rightarrow \mathbb{R}$ is a continuous and differentiable function satisfying (A1) and $(A 2)$. Let $v_{0}, v \in L^{\infty}(\Omega)$ belonging to $\dot{V}_{+}^{r} \stackrel{\text { def }}{=}\left\{v: \Omega \rightarrow(0,+\infty) \mid v^{\frac{1}{r}} \in W_{0}^{1, p(x)}(\Omega)\right\}$ for some $r \geq 1$. Then

$$
\frac{1}{p(x)}\left\langle\partial_{\xi} A\left(x, \nabla v_{0}^{1 / r}\right), \nabla\left(\frac{v}{v_{0}^{(r-1) / r}}\right)\right\rangle \leq A^{\frac{r}{p(x)}}\left(x, \nabla v^{1 / r}\right) A^{\frac{(p(x)-r)}{p(x)}}\left(x, \nabla v_{0}^{1 / r}\right)
$$

where $\langle.,$.$\rangle is the inner scalar product and the above inequality is strict if r>1$ or $\frac{v}{v_{0}} \not \equiv$ Const $>0$.

From the above Picone identity, we can show an extension of the famous Diaz-Saa inequality to the class of operators with variable exponent. This inequality is strongly linked to the strict convexity of some associated homogeneous energy type functional.

Theorem 1.2 (Diaz-Saa inequality). Let $A: \Omega \times \mathbb{R}^{N} \rightarrow \mathbb{R}$ is a continuous and differentiable function satisfying $(A 1)$ and $(A 2)$ and define $a(x, \xi)=\left(a_{i}(x, \xi)\right)_{i} \stackrel{\text { def }}{=}\left(\frac{1}{p(x)} \partial_{\xi_{i}} A(x, \xi)\right)_{i}$. Assume in addition that there exists $\Lambda>0$ such that

$$
a \in C^{1}\left(\Omega \times\left(\mathbb{R}^{N} \backslash\{0\}\right)\right)^{N} \text { and } \sum_{i, j=1}^{N}\left|\frac{\partial a_{i}(x, \xi)}{\partial \xi_{j}}\right| \leq \Lambda|\xi|^{p(x)-2}
$$

for all $(x, \xi) \in \Omega \times \mathbb{R}^{N} \backslash\{0\}$. Then, we have in the sense of distributions, for any $r \in\left[1, p_{-}\right]$

$$
\int_{\Omega}\left(-\frac{\operatorname{div}\left(a\left(x, \nabla w_{1}\right)\right)}{w_{1}^{r-1}}+\frac{\operatorname{div}\left(a\left(x, \nabla w_{2}\right)\right)}{w_{2}^{r-1}}\right)\left(w_{1}^{r}-w_{2}^{r}\right) d x \geq 0
$$


for any $w_{1}, w_{2} \in W_{0}^{1, p(x)}(\Omega)$, positive in $\Omega$ such that $\frac{w_{1}}{w_{2}}, \frac{w_{2}}{w_{1}} \in L^{\infty}(\Omega)$. Moreover, if the equality occurs in (1.6), then $w_{1} / w_{2}$ is constant in $\Omega$. If $p(x) \not \equiv$ in $\Omega$ then even $w_{1}=w_{2}$ holds in $\Omega$.

In sections 3, 4 and 5, we derive some applications of the new Picone identity. Precisely, we investigate the solvability of some boundary problems involving quasilinear elliptic operators with variable exponent. In section 3, we consider the following nonlinear problem:

$$
\left\{\begin{aligned}
-\Delta_{p(x)} u+g(x, u) & =f(x, u) & & \text { in } \Omega ; \\
u & >0 & & \text { in } \Omega ; \\
u & =0 & & \text { on } \partial \Omega .
\end{aligned}\right.
$$

The extended Picone identity can be reformulated as in Lemma 3.1 below. Together with the strong maximum principle and elliptic regularity, this identity can be used to prove the uniqueness of weak solutions to elliptic equations as (1.7). In particular, we establish the following result:

Theorem 1.3. Let $f, g: \Omega \times[0, \infty) \rightarrow \mathbb{R}^{+}$be defined as $f(x, t)=h(x) t^{q(x)-1}$ and $g(x, t)=l(x) t^{s(x)-1}$ with $1 \leq q, s \in C(\bar{\Omega})$ such that

- $\quad q_{+}<p_{-}<s_{-}$and $q_{-} \geq 1$;

- $\quad h, l \in L^{\infty}(\Omega)$, positive functions such that $x \rightarrow \frac{h(x)}{l(x)} \in L^{\infty}(\Omega)$.

Then, there exists a weak solution $u$ to (1.7), i.e. $u$ belongs to $W_{0}^{1, p(x)}(\Omega) \cap L^{s(x)}(\Omega)$ and satisfies for any $\phi \in$ $W_{0}^{1, p(x)}(\Omega) \cap L^{s(x)}(\Omega):$

$$
\int_{\Omega}|\nabla u|^{p(x)-2} \nabla u . \nabla \phi d x=\int_{\Omega}(f(x, u)-g(x, u)) \phi d x .
$$

Furthermore $u \in C^{1, \alpha}(\bar{\Omega})$ for some $\alpha \in(0,1)$ and $0 \leq u^{s_{-}-q_{+}} \leq \max \left\{\left\|\frac{h}{l}\right\|_{L^{\infty}}, 1\right\}$ a.e. in $\Omega$.

Assume in addition that $x \rightarrow \frac{l(x)}{h(x)}$ belongs to $L^{\infty}(\Omega)$, then $u \in C_{d}^{0}(\bar{\Omega})^{+} \stackrel{\text { def }}{=}\left\{v \in C_{0}(\bar{\Omega}) \mid \exists c_{1}, c_{2} \in \mathbb{R}_{\star}^{+}: c_{1} \leq\right.$ $\left.\frac{v}{\operatorname{dist}(x, \partial \Omega)} \leq c_{2}\right\}$ and is the unique weak solution to (1.7).

Regarding the current literature, Theorem 1.3 does not require any subcritical growth condition for $g$ to establish existence and uniqueness of the weak solution to (1.7).

In section 4, we study a nonlinear fast diffusion equation (F.D.E. for short) driven by $p(x)$-Laplacian. From the physical Fick's law, the diffusion coefficient of our problem is then proportional to $|\nabla u(x, t)|^{p(x)-2}$. It naturally leads to investigate the following F.D.E. type problem:

$$
\left\{\begin{aligned}
\frac{q}{2 q-1} \partial_{t}\left(u^{2 q-1}\right)-\Delta_{p(x)} u & =f(x, u)+h(t, x) u^{q-1} & & \text { in } Q_{T} ; \\
u & >0 & & \text { in } Q_{T} ; \\
u & =0 & & \text { on } \Gamma ; \\
u(0, .) & =u_{0} & & \text { in } \Omega
\end{aligned}\right.
$$

where $q \in\left(1, p_{-}\right), Q_{T}=(0, T) \times \Omega$ and $\Gamma=(0, T) \times \partial \Omega$ for some $T>0$. We suppose that $h \in L^{\infty}\left(Q_{T}\right)$ and nonnegative. The assumptions on $f$ are given by

$\left(f_{1}\right) \quad f: \Omega \times \mathbb{R}^{+} \rightarrow \mathbb{R}^{+}$is a function such that $f(x, 0)=0$ for all $x \in \Omega$ and $f \neq 0$;

$\left(f_{2}\right) \quad \lim _{s \rightarrow 0^{+}} \frac{f(x, s)}{s^{2 q-1}}=+\infty$ uniformly in $x$;

$\left(f_{3}\right) \quad$ for any $x \in \Omega, s \rightarrow \frac{f(x, s)}{s^{q-1}}$ is nonincreasing in $\mathbb{R}^{+} \backslash\{0\}$. 
Remark 1.2. Conditions $\left(f_{1}\right)$ and $\left(f_{3}\right)$ imply there exist positive constant $C_{1}, C_{2}$ such that for any $(x, s) \in \Omega \times \mathbb{R}^{+}$:

$$
0 \leq f(x, s) \leq C_{1}+C_{2} s^{q-1}
$$

i.e. f has a strict subhomogeneous growth.

We set $\mathcal{R}$ the operator defined by $\mathcal{R} v=\frac{-\Delta_{p(x)}\left(v^{1 / q}\right)}{v^{(q-1) / q}}-\frac{f\left(x, v^{1 / q}\right)}{v^{(q-1) / q}}$ and the associated domain

$$
\mathcal{D}(\mathcal{R})=\left\{v: \Omega \rightarrow(0, \infty): v^{1 / q} \in W_{0}^{1, p(x)}(\Omega), v \in L^{2}(\Omega), \mathcal{R} v \in L^{2}(\Omega)\right\}
$$

Note that $\mathcal{D}(\mathcal{R})$ contains for instance solutions to (4.18). One can also easily check that solutions to (4.19) belong to $\overline{\mathcal{D}(\mathcal{R})}{ }^{L^{2}(\Omega)}$. In the sequel, we denote $X^{+} \stackrel{\text { def }}{=}\{x \in X \mid x \geq 0\}$ the associated positive cone of a given real vector space $X$.

In order to establish existence and properties of weak solutions to (1.8), we investigate the following related parabolic problem:

$$
\left\{\begin{aligned}
v^{q-1} \partial_{t}\left(v^{q}\right)-\Delta_{p(x)} v & =h(t, x) v^{q-1}+f(x, v) & & \text { in } Q_{T} ; \\
v & >0 & & \text { in } Q_{T} ; \\
v & =0 & & \text { on } \Gamma ; \\
v(0, .) & =v_{0}(x)>0 & & \text { in } \Omega .
\end{aligned}\right.
$$

The notion of weak solution for (1.9) is given as follows:

Definition 1.1. A weak solution to (1.9) is any positive function $v \in L^{\infty}\left(0, T ; W_{0}^{1, p(x)}(\Omega)\right) \cap L^{\infty}\left(Q_{T}\right) \cap C\left(0, T ; L^{r}(\Omega)\right)$ for any $r \geq 1$ such that $\partial_{t}\left(v^{q}\right) \in L^{2}\left(Q_{T}\right)$ and for any $\phi \in C_{0}^{\infty}\left(Q_{T}\right)$ satisfies

$$
\int_{0}^{T} \int_{\Omega} \partial_{t}\left(v^{q}\right) v^{q-1} \phi d x d t+\int_{0}^{T} \int_{\Omega}|\nabla v|^{p(x)-2} \nabla v \cdot \nabla \phi d x d t=\int_{0}^{T} \int_{\Omega} h(t, x) v^{q-1} \phi d x d t+\int_{0}^{T} \int_{\Omega} f(x, v) \phi d x d t
$$

Concerning (1.9), we prove the following results:

Theorem 1.4. Let $T>0, v_{0} \in C_{d}^{0}(\bar{\Omega})^{+} \cap W_{0}^{1, p(x)}(\Omega)$. In addition, there exists $h_{0} \in L^{\infty}(\Omega), h_{0} \not \equiv 0$ and $h(t, x) \geq$ $h_{0}(x) \geq 0$ for a.e $x \in \Omega$, for a.e. $t \geq 0$. Assume in addition $q \leq \min \left\{\frac{N}{2}+1, p_{-}\right\}$and $f$ satisfies $\left(f_{1}\right)-\left(f_{3}\right)$. Then there exists a weak solution to (1.9).

Based on the accretivity of $\mathcal{R}$ with domain $\mathcal{D}(\mathcal{R})$, we show the following result providing a contraction property for weak solutions to (1.9) under suitable conditions on initial data:

Theorem 1.5. Let $v_{1}$ and $v_{2}$ are weak solutions of (1.9) with initial data $u_{0}, v_{0} \in C_{d}^{0}(\bar{\Omega})^{+} \cap W_{0}^{1, p(x)}(\Omega)$ and such that $u_{0}^{q}, v_{0}^{q} \in \overline{\mathcal{D}(\mathcal{R})}^{L^{2}(\Omega)}$ and $h, g \in L^{\infty}\left(Q_{T}\right)$, such that $h \geq h_{0}, g \geq g_{0}$ with $h_{0}, g_{0}$ as in Theorem 1.4. Then, for any $0 \leq t \leq T$,

$$
\left\|\left(v_{1}^{q}(t)-v_{2}^{q}(t)\right)^{+}\right\|_{L^{2}} \leq\left\|\left(u_{0}^{q}-v_{0}^{q}\right)^{+}\right\|_{L^{2}}+\int_{0}^{t}\left\|(h(s)-g(s))^{+}\right\|_{L^{2}} d s .
$$

Furthermore, using a similar approach as in [8], we consider for $\epsilon>0$ the perturbed operator $\mathcal{R}_{\epsilon} v=$ $\frac{-\Delta_{p(x)}\left(v^{1 / q}\right)}{(v+\epsilon)^{(q-1) / q}}-\frac{f\left(x, v^{1 / q}\right)}{(v+\epsilon)^{(q-1) / q}}$. If $p_{-} \geq 2$, we can prove (as in Proposition 2.6 in [8]) that

$$
{\overline{\mathcal{D}\left(\mathcal{R}_{\epsilon}\right)}}^{L^{2}(\Omega)} \supset \dot{V}_{+}^{q} \cap C_{d^{q}}^{0}(\bar{\Omega})^{+}
$$

Arguing as in Theorem 1.5 with the operator $\mathcal{R}_{\epsilon}$ instead of $\mathcal{R}$ and passing to the limit as $\epsilon \rightarrow 0^{+}$, we get: 
Corollary 1.1. Assume $p_{-} \geq 2$. Let $v_{1}$ and $v_{2}$ are weak solutions of (1.9) with initial data $u_{0}, v_{0} \in C_{d}^{0}(\bar{\Omega})^{+} \cap$ $W_{0}^{1, p(x)}(\Omega)$. Then Theorem 1.5 holds.

From Theorem 1.5, we derive the following comparison principle from which uniqueness of the weak solution to problem (1.9) follows:

Corollary 1.2. Let $u$ and $v$ are the weak solutions of (1.9) with initial data $u_{0}, v_{0}$ satisfying conditions in Theorem 1.5 or Corollary 1.1. Assume $u_{0} \leq v_{0}$ and $h, g \in L^{\infty}\left(Q_{T}\right), h_{0} \in L^{\infty}(\Omega)$ such that and $0<h_{0} \leq h \leq g$. Then $u \leq v$.

Remark 1.3. If $v \in L^{\infty}\left(Q_{T}\right)^{+}$then from Proposition 9.5 in [18] we obtain $\frac{q}{2 q-1} \partial_{t}\left(v^{2 q-1}\right)=v^{q-1} \partial_{t}\left(v^{q}\right)=$ $q v^{2 q-2} \partial_{t} v$ in weak sense.

Remark 1.4. From Theorem 1.5, we can derive stabilization results for the evolution equation $(1.8)$ in $L^{q}(\Omega)$ with $q \in[2, \infty)$ (see [12] in this regard).

From the above remark, under assumptions given in Theorem 1.4, we obtain the existence of weak solutions to (1.8) satisfying the monotonicity properties in Theorem 1.5 and Corollaries 1.1,1.2. We highlight that in our knowledge there is no result available in the current literature about F.D.E. with variable exponent. In this regard our results are completely new.

In the previous applications, the condition $(A 1)$ plays a crucial role to get suitable convexity property of energy functionals. In section 5, we study a quasilinear elliptic problem where this condition is not satisfied. Precisely, given $\epsilon>0$, we study the following nonhomogeneous quasilinear elliptic problem:

$$
\left\{\begin{aligned}
-\operatorname{div}\left(\left(|\nabla u|^{2}+\epsilon u^{2}\right)^{\frac{p(x)-2}{2}} \nabla u\right)-\left(|\nabla u|^{2}+\epsilon u^{2}\right)^{\frac{p(x)-2}{2}} \epsilon u & =g(x, u) & & \text { in } \Omega ; \\
u & =0 & & \text { on } \partial \Omega ; \\
u & >0 & & \text { in } \Omega
\end{aligned}\right.
$$

where $g$ satisfies $\left(f_{1}\right)$ and $(\tilde{g})$ for some $m \in\left[1, p_{-}\right]$:

( $\tilde{g}) \quad$ For any $x \in \Omega, s \rightarrow \frac{g(x, s)}{s^{m-1}}$ is decreasing in $\mathbb{R}^{+} \backslash\{0\}$ and a.e. in $\Omega$.

Then we prove the following result:

Theorem 1.6. Assume that $g$ satisfies $\left(f_{1}\right)$ and $(\tilde{g})$. Then for any $\epsilon,(1.12)$ admits one and only one positive weak solution. Furthermore, $u \in C^{1}(\bar{\Omega}), u>0$ in $\Omega$ and $\frac{\partial u}{\partial \vec{n}}<0$ on $\partial \Omega$.

To get the uniqueness result contained in Theorem 1.6, we exploit the hidden convexity property of the associated energy functional in the interior of positive cone of $C^{1}(\bar{\Omega})$.

\section{Picone identity and Diaz-Saa inequality}

\subsection{Picone identity}

First we recall the notion of strict ray-convexity.

Definition 2.1. Let $X$ be a real vector space. Let $\dot{V}$ be a non empty cone in $X$. A function $J: \dot{V} \rightarrow \mathbb{R}$ is ray-strictly convex if for all $v_{1}, v_{2} \in \dot{V}$ and for all $\theta \in(0,1)$

$$
J\left((1-\theta) v_{1}+\theta v_{2}\right) \leq(1-\theta) J\left(v_{1}\right)+\theta J\left(v_{2}\right)
$$


where the inequality is always strict unless $v_{1}=C v_{2}$ for some $C>0$.

Then we have the following result:

Proposition 2.1. Let $A$ satisfying $(A 1)$ and $(A 2)$ and let $r \geq 1$. Then, for any $x \in \Omega$ the map $\xi \rightarrow N_{r}(x, \xi) \stackrel{\text { def }}{=}$ $A(x, \xi)^{r / p(x)}$ is positively $r$-homogeneous and ray-strictly convex. For $r>1, \xi \rightarrow N_{r}(x, \xi)$ is even strictly convex.

Proof. We begin by the case $r=1$. For any $t \in \mathbb{R}^{+}$, we have $N_{1}(x, t \xi)=t N_{1}(x, \xi)$. Furthermore,

$$
A\left(x,(1-t) \xi_{1}+t \xi_{2}\right) \leq(1-t) A\left(x, \xi_{1}\right)+t A\left(x, \xi_{2}\right) \leq \max \left\{A\left(x, \xi_{1}\right), A\left(x, \xi_{2}\right)\right\}
$$

for any $x \in \Omega, \xi_{1}, \xi_{2} \in \mathbb{R}^{N}$ and $t \in[0,1]$. Therefore

$$
N_{1}\left(x,(1-t) \xi_{1}+t \xi_{2}\right) \leq \max \left\{N_{1}\left(x, \xi_{1}\right), N_{1}\left(x, \xi_{2}\right)\right\}
$$

and this inequality is always strict unless $\xi_{1}=\lambda \xi_{2}$, for some $\lambda>0$.

Now we prove that $N_{1}$ is subadditive.

Without loss of generality, we can assume that $\xi_{1} \neq 0$ and $\xi_{2} \neq 0$. Then we have $N_{1}\left(x, \xi_{1}\right)>0$ and $N_{1}\left(x, \xi_{2}\right)>$ 0 . Therefore, from (2.1) and 1-homogeneity of $N_{1}(x, \xi)$ we obtain for any $t \in(0,1)$ :

$$
N_{1}\left(x,(1-t) \frac{\xi_{1}}{N_{1}\left(x, \xi_{1}\right)}+t \frac{\xi_{2}}{N_{1}\left(x, \xi_{2}\right)}\right) \leq 1
$$

We now fix $t$ such that

$$
\frac{1-t}{N_{1}\left(x, \xi_{1}\right)}=\frac{t}{N_{1}\left(x, \xi_{2}\right)} \text { i.e. } t=\frac{N_{1}\left(x, \xi_{2}\right)}{N_{1}\left(x, \xi_{1}\right)+N_{1}\left(x, \xi_{2}\right)} \leq 1 \text {. }
$$

Then we get

$$
N_{1}\left(x, \frac{\xi_{1}+\xi_{2}}{N_{1}\left(x, \xi_{1}\right)+N_{1}\left(x, \xi_{2}\right)}\right) \leq 1
$$

and by 1-homogeneity of $N_{1}$, we obtain

$$
N_{1}\left(x, \xi_{1}+\xi_{2}\right) \leq N_{1}\left(x, \xi_{1}\right)+N_{1}\left(x, \xi_{2}\right) \text {, i.e. } N_{1} \text { is subadditive. }
$$

Finally for $t \in(0,1), \xi_{1} \neq \lambda \xi_{2}, \forall \lambda>0$

$$
N_{1}\left(x,(1-t) \xi_{1}+t \xi_{2}\right)<N_{1}\left(x,(1-t) \xi_{1}\right)+N_{1}\left(x, t \xi_{2}\right)=(1-t) N_{1}\left(x, \xi_{1}\right)+t N_{1}\left(x, \xi_{2}\right) .
$$

This proves that $\xi \rightarrow N_{1}(x, \xi)$ is ray-strictly convex. Now consider the case $r>1$. Since for any $x \in \Omega, \xi \rightarrow$ $N_{r}^{1 / r}(x, \xi)=N_{1}(x, \xi)$ is ray-strictly convex and thanks to the strict convexity of $t \rightarrow t^{r}$ on $\mathbb{R}^{+}$, we deduce that $\xi \rightarrow N_{r}(x, \xi)=N_{1}^{r}(x, \xi)$ is strictly convex when $r>1$.

From Proposition 2.1 and from the $r$-homogeneity of $N_{r}$, we easily deduce the following convexity property of the energy functional:

Proposition 2.2. Under hypothesis of Proposition 2.1 and assume in addition $A$ is continuous on $\bar{\Omega} \times \mathbb{R}^{N}$. Then, for $1 \leq r<p_{-}$:

$$
\dot{V}_{+}^{r} \cap L^{\infty}(\Omega) \ni v \rightarrow \int_{\Omega} A\left(x, \nabla\left(v^{1 / r}\right)\right) d x
$$

is ray-strictly convex (if $r>1$, it is even strictly convex).

Proof. We know that $\xi \rightarrow N_{r}(x, \xi)=A^{r / p(x)}(x, \xi)$ is $r$-positively homogeneous and strictly convex if $r>1$ and for $r=1$ this function is ray-strictly convex. For $v_{1}, v_{2} \in \dot{V}_{+}^{r}$ and $\theta \in(0,1)$ define $v=(1-\theta) v_{1}+\theta v_{2}$ and we get

$$
N_{r}\left(x, \frac{\nabla v}{v}\right) \leq(1-\theta) \frac{v_{1}}{v} N_{r}\left(x, \frac{\nabla v_{1}}{v_{1}}\right)+\theta \frac{v_{2}}{v} N_{r}\left(x, \frac{\nabla v_{2}}{v_{2}}\right) .
$$


By homogeneity,

$$
N_{r}\left(x, \nabla\left(v^{1 / r}\right)\right) \leq(1-\theta) N_{r}\left(x, \nabla\left(v_{1}^{1 / r}\right)\right)+\theta N_{r}\left(x, \nabla\left(v_{2}^{1 / r}\right)\right)
$$

and equality holds if and only if $v_{1}=\lambda v_{2}$ for some $\lambda>0$. Using the convexity of $t \rightarrow t^{p(x) / r}$ for $1 \leq r<p$ - we obtain

$$
\int_{\Omega} A\left(x, \nabla v^{1 / r}\right) d x \leq(1-\theta) \int_{\Omega} A\left(x, \nabla v_{1}^{1 / r}\right) d x+\theta \int_{\Omega} A\left(x, \nabla v_{2}^{1 / r}\right) d x .
$$

Moreover, if $p(x) \neq r$ equality holds if and only if $v_{1}=v_{2}$.

From Proposition 2.1, we deduce the proof of Picone identity.

Proof of Theorem 1.1: Firstly, we deal with the case $r>1$. Then from Proposition 2.1, for any $x \in \Omega$ the function $\xi \rightarrow N_{r}(x, \xi)=A(x, \xi)^{r / p(x)}$ is strictly convex. Let $\xi, \xi_{0} \in \mathbb{R}^{N} \backslash\{0\}$ such that $\xi \neq \xi_{0}$ then

$$
N_{r}(x, \xi)-N_{r}\left(x, \xi_{0}\right)>\left\langle\partial_{\xi} N_{r}\left(x, \xi_{0}\right), \xi-\xi_{0}\right\rangle .
$$

Setting $\tilde{a}(x, \xi)=\frac{1}{r} \partial_{\xi} N_{r}(x, \xi)$, we obtain:

$$
N_{r}(x, \xi)-\left\langle\tilde{a}\left(x, \xi_{0}\right), \xi_{0}\right\rangle>r\left\langle\tilde{a}\left(x, \xi_{0}\right), \xi-\xi_{0}\right\rangle .
$$

Let $v, v_{0}>0$ and replacing $\xi, \xi_{0}$ by $\xi / v$ and $\xi_{0} / v_{0}$ respectively in the above expression, we get

$$
N_{r}\left(x, \frac{\xi}{v}\right)>r\left\langle\tilde{a}\left(x, \frac{\xi_{0}}{v_{0}}\right), \frac{\xi}{v}-\frac{r-1}{r} \frac{\xi_{0}}{v_{0}}\right\rangle .
$$

Taking $\xi=\nabla v$ and $\xi_{0}=\nabla v_{0}$ and using $(r-1)$-homogeneity of $\tilde{a}(x,$.$) ,$

$$
N\left(x, \frac{\nabla v}{r v^{(r-1) / r}}\right)>\frac{1}{v_{0}^{(r-1) / r}}\left\langle\tilde{a}\left(x, \frac{\nabla v_{0}}{r v_{0}^{(r-1) / r}}\right), \nabla v-\frac{r-1}{r} \frac{\nabla v_{0}}{v_{0}} v\right\rangle
$$

where the inequality is strict unless $\frac{\nabla v}{v}=\frac{\nabla v_{0}}{v_{0}}$.

Since $v^{1 / r}, v_{0}^{1 / r} \in W^{1, p(x)}(\Omega) \cap L^{\infty}(\Omega)$, we can write

$$
\nabla\left(v^{1 / r}\right)=\frac{\nabla v}{r v^{(r-1) / r}} \quad \text { and } \quad \nabla\left(\frac{v}{v_{0}^{(r-1) / r}}\right)=\frac{1}{v_{0}^{(r-1) / r}}\left(\nabla v-\frac{r-1}{r} \frac{\nabla v_{0}}{v_{0}} v\right)
$$

and we obtain

$$
N\left(x, \nabla v^{1 / r}\right)>\left\langle\tilde{a}\left(x, \nabla v_{0}^{1 / r}\right), \nabla\left(\frac{v}{v_{0}^{(r-1) / r}}\right)\right\rangle .
$$

We have

$$
\begin{aligned}
\tilde{a}\left(x, \nabla v_{0}^{1 / r}\right) & =\frac{1}{r} \partial_{\xi} N\left(x, \nabla v_{0}^{1 / r}\right)=\frac{1}{r} \partial_{\xi} A^{r / p(x)}\left(x, \nabla v_{0}^{1 / r}\right) \\
& =\frac{1}{p(x)} \partial_{\xi} A\left(x, \nabla v_{0}^{1 / r}\right) A^{\frac{r-p(x)}{p(x)}}\left(x, \nabla v_{0}^{1 / r}\right)
\end{aligned}
$$

and by replacing in (2.2) we obtain

$$
A^{\frac{r}{p(x)}}\left(x, \nabla v^{1 / r}\right) A^{\frac{p(x)-r}{p(x)}}\left(x, \nabla v_{0}^{1 / r}\right)>\frac{1}{p(x)}\left\langle\partial_{\xi} A\left(x, \nabla v_{0}^{1 / r}\right), \nabla\left(\frac{v}{v_{0}^{(r-1) / r}}\right)\right\rangle .
$$

Now we deal with the case $r=1$. Let $\xi, \xi_{0} \in \mathbb{R}^{N} \backslash\{0\}$ such that for any $\lambda>0, \xi \neq \lambda \xi_{0}$. Then, from Proposition 2.1, we have that

$$
N(x, \xi)-N\left(x, \xi_{0}\right) \geq\left\langle\partial_{\xi} N\left(x, \xi_{0}\right), \xi-\xi_{0}\right\rangle .
$$

Taking $\xi=\nabla v$ and $\xi_{0}=\nabla v_{0}$, we deduce

$$
N(x, \nabla v)-N\left(x, \nabla v_{0}\right) \geq\left\langle\partial_{\xi} N\left(x, \nabla v_{0}\right), \nabla\left(v-v_{0}\right)\right\rangle
$$


and

$$
A^{\frac{1}{p(x)}}(x, \nabla v) A^{\frac{p(x)-1}{p(x)}}\left(x, \nabla v_{0}\right) \geq \frac{1}{p(x)}\left\langle\partial_{\xi} A\left(x, \nabla v_{0}\right), \nabla v\right\rangle
$$

for any $x \in \Omega$ and the inequality is strict unless $v=\lambda v_{0}$ for some $\lambda>0$.

The Picone identity also holds for anisotropic operators of the following type:

$$
\sum_{i=1}^{N} \nabla_{i}\left(b_{i}\left(x, \nabla_{i} u\right)\right)=\sum_{i=1}^{N} \frac{\partial}{\partial x_{i}}\left(b_{i}\left(x, \frac{\partial u}{\partial x_{i}}\right)\right) .
$$

Precisely we have:

Corollary 2.1. Let $B: \Omega \times \mathbb{R} \rightarrow \mathbb{R}^{N}$ is a continuous and differentiable function such that $B(x, s)=$ $\left(B_{i}(x, s)\right)_{i=1,2, \ldots N}$ satisfying for any $i$, for any $x \in \Omega$, the map $s \rightarrow B_{i}(x, s)$ is $p_{i}(x)$-homogeneous and strictly convex with $1<p_{i}^{-} \leq p_{i}(\cdot) \leq p_{i}^{+}<\infty$. For any $i$, we define $b_{i}(x, s)=\frac{1}{p_{i}(x)} \partial_{s} B_{i}(x, s)$. Then, for $v, v_{0} \in \dot{V}_{+}^{r} \cap L^{\infty}(\Omega)$, we have

$$
\sum_{i=1}^{N} b_{i}\left(x, \partial_{x_{i}}\left(v_{0}^{1 / r}\right)\right) \partial_{x_{i}}\left(\frac{v}{v_{0}^{\frac{r-1}{r}}}\right) \leq \sum_{i=1}^{N} B_{i}^{\frac{r}{p_{i}(x)}}\left(x, \partial_{x_{i}}\left(v^{1 / r}\right)\right) B_{i}^{\frac{p_{i}(x)-r}{p_{i}(x)}}\left(x, \partial_{x_{i}}\left(v_{0}^{1 / r}\right)\right) .
$$

Proof. By taking $A(x, s)=B_{i}(x, s)$ in Theorem 1.1, we obtain $\forall i \in\{1,2, \ldots, N\}$

$$
\frac{1}{p_{i}(x)} \partial_{s} B_{i}\left(x, \partial_{x_{i}}\left(v_{0}^{1 / r}\right)\right) \cdot \partial_{x_{i}}\left(\frac{v}{v_{0}^{\frac{r-1}{r}}}\right) \leq B_{i}^{\frac{r}{p_{i}(x)}}\left(x, \partial_{x_{i}}\left(v^{1 / r}\right)\right) \cdot B_{i}^{\frac{p_{i}(x)-r}{p_{i}(x)}}\left(x, \partial_{x_{i}}\left(v_{0}^{1 / r}\right)\right)
$$

for all $v, v_{0} \in \dot{V}_{+}^{r} \cap L^{\infty}(\Omega)$ and $i=1,2, \ldots, N$.

Then by summing the expression over $i=1,2, \ldots, N$, we obtain

$$
\sum_{i=1}^{N} b_{i}\left(x, \partial_{x_{i}}\left(v_{0}^{1 / r}\right)\right) \cdot \partial_{x_{i}}\left(\frac{v}{v_{0}^{r-1}}\right) \leq \sum_{i=1}^{N} B_{i}^{\frac{r}{p_{i}(x)}}\left(x, \partial_{x_{i}}\left(v^{1 / r}\right)\right) \cdot B_{i}^{\frac{p_{i}(x)-r}{p_{i}(x)}}\left(x, \partial_{x_{i}}\left(v_{0}^{1 / r}\right)\right) .
$$

\subsection{An extension of the Diaz-Saa inequality}

We prove the first application of Picone identity.

Proof of Theorem 1.2: The Picone identity implies

$$
A^{r / p(x)}\left(x, \nabla w_{1}\right) A^{(p(x)-r) / p(x)}\left(x, \nabla w_{2}\right) \geq a\left(x, \nabla w_{2}\right) . \nabla\left(\frac{w_{1}^{r}}{w_{2}^{r-1}}\right) .
$$

Using the Young inequality for $r \in\left[1, p_{-}\right]$, we get

$$
\frac{r}{p(x)}\left(A\left(x, \nabla w_{1}\right)-A\left(x, \nabla w_{2}\right)\right)+A\left(x, \nabla w_{2}\right) \geq a\left(x, \nabla w_{2}\right) . \nabla\left(\frac{w_{1}^{r}}{w_{2}^{r-1}}\right) .
$$

Noting that for any $\xi \in \mathbb{R}^{N}, A(x, \xi)=a(x, \xi) . \xi$, we deduce

$$
a\left(x, \nabla w_{2}\right) . \nabla\left(w_{2}-\frac{w_{1}^{r}}{w_{2}^{r-1}}\right) d x \geq \frac{r}{p(x)}\left(A\left(x, \nabla w_{2}\right)-A\left(x, \nabla w_{1}\right)\right) .
$$

Commuting $w_{1}$ and $w_{2}$, we have

$$
a\left(x, \nabla w_{1}\right) . \nabla\left(w_{1}-\frac{w_{2}^{r}}{w_{1}^{r-1}}\right) \geq \frac{r}{p(x)}\left(A\left(x, \nabla w_{1}\right)-A\left(x, \nabla w_{2}\right)\right) .
$$


Summing (2.3) and (2.4) and integrating over $\Omega$ yield

$$
\int_{\Omega} a\left(x, \nabla w_{1}\right) \cdot \nabla\left(\frac{w_{1}^{r}-w_{2}^{r}}{w_{1}^{r-1}}\right) d x+\int_{\Omega} a\left(x, \nabla w_{2}\right) \cdot \nabla\left(\frac{w_{2}^{r}-w_{1}^{r}}{w_{2}^{r-1}}\right) \geq 0 .
$$

The rest of the proof is the consequence of Proposition 2.2.

Diaz-Saa inequality also holds for anisotropic operators. Here we require that $\xi \rightarrow B_{i}(x, \xi)$ is $p_{i}(x)$ homogeneous and strictly convex and $b_{i}(x, \xi)=\frac{1}{p_{i}(x)} \partial_{i} B_{i}(x, \xi)$ where $r \in \mathbb{R}, 1 \leq r \leq \min _{i=1,2, \ldots, N}\left\{\left(p_{i}\right)_{-}\right\}$.

Corollary 2.2. Under the assumptions of Corollary 2.1 and in addition that there exist $\Lambda>0$ such that for each $i,\left|\frac{\partial b_{i}}{\partial s}(x, s)\right| \leq \Lambda|s|^{p(x)-2}$. Then we have in the sense of distributions, for $r \in\left[1, \min _{i}\left\{\left(p_{i}\right)_{-}\right\}\right]$and $v, v_{0} \in$ $\dot{V}_{+}^{r} \cap L^{\infty}(\Omega):$

$$
\sum_{i=1}^{N} \int_{\Omega}\left(-\frac{\partial_{x_{i}}\left(b_{i}\left(x, \partial_{x_{i}} v\right)\right)}{v^{r-1}(x)}+\frac{\partial_{x_{i}}\left(b_{i}\left(x, \partial_{x_{i}} v_{0}\right)\right)}{v_{0}^{r-1}(x)}\right)\left(v^{r}-v_{0}^{r}\right) d x \geq 0
$$

Proof. We apply Theorem 1.2. For $A=B_{i}: \Omega \times \mathbb{R} \rightarrow \mathbb{R}$ and by replacing $\nabla$ by $\partial_{x_{i}}$.

\section{Application of Picone identity to quasilinear elliptic equations}

The aim of this section is to establish Theorem 1.3.

\subsection{Preliminary results}

The first lemma is the Picone identity in the context of the $p(x)$-Laplacian operator.

Lemma 3.1. Let $r \in\left[1, p_{-}\right]$and $u, v \in W_{0}^{1, p(x)}(\Omega) \cap L^{\infty}(\Omega)$ two positive functions. Then for any $x \in \Omega$

$$
|\nabla u|^{p(x)}+|\nabla v|^{p(x)} \geq|\nabla v|^{p(x)-2} \nabla v \cdot \nabla\left(\frac{u^{r}}{v^{r-1}}\right)+|\nabla u|^{p(x)-2} \nabla u \cdot \nabla\left(\frac{v^{r}}{u^{r-1}}\right) .
$$

Following the proof of Theorem 1.1 in [19], we first prove the following comparison principle:

Lemma 3.2. Let $\lambda \geq 0$ and $u, v \in W_{0}^{1, p(x)}(\Omega) \cap L^{\alpha(x)}(\Omega)$ two nonnegative functions for some function $\alpha \in \mathcal{P}(\Omega)$ satisfying $1<\alpha_{-} \leq \alpha_{+}<\infty$. Assume for any $\phi \in W_{0}^{1, p(x)}(\Omega), \phi \geq 0$ :

$$
\int_{\Omega}|\nabla u|^{p(x)-2} \nabla u \cdot \nabla \phi+u^{\alpha(x)-1} \chi_{u \geq \lambda} \phi d x \geq \int_{\Omega}|\nabla v|^{p(x)-2} \nabla v \cdot \nabla \phi+v^{\alpha(x)-1} \chi_{v \geq \lambda} \phi d x
$$

where

$$
\chi_{v \geq \lambda}(x)= \begin{cases}1 & \text { if } \lambda \leq v<\infty \\ 0 & \text { if } 0 \leq v<\lambda\end{cases}
$$

and $u \geq v$ a.e. in $\partial \Omega$. Then $u \geq v$ a.e. in $\Omega$.

Proof. Let $\phi=(v-u)^{+} \in W_{0}^{1, p(x)}(\Omega)$ and $\Omega_{1}=\{x \in \Omega: u(x)<v(x)\}$. Then

$$
0 \leq-\int_{\Omega_{1}}\left(|\nabla u|^{p(x)-2} \nabla u-|\nabla v|^{p(x)-2} \nabla v\right) . \nabla(u-v) d x-\int_{\Omega_{1}}\left(u^{\alpha(x)-1} \chi_{u \geq \lambda}-v^{\alpha(x)-1} \chi_{v \geq \lambda}\right)(u-v) d x \leq 0
$$

from which we obtain $u \geq v$ a.e. in $\Omega$. 
Using lemma 3.2, we show the following strong maximum principle:

Lemma 3.3. Let $h, l \in L^{\infty}(\Omega)$ be nonnegative functions, $h>0$ and $k: \Omega \times \mathbb{R}^{+} \rightarrow \mathbb{R}^{+}$. Let $\alpha, \beta \in \mathcal{P}(\Omega)$ be two functions such that $1<\beta_{-} \leq \beta_{+}<\alpha_{-} \leq \alpha_{+}<\infty$. Let $u \in C^{1}(\bar{\Omega})$ be nonnegative and a nontrivial solution to

$$
\left\{\begin{aligned}
-\Delta_{p(x)} u+l(x) u^{\alpha(x)-1} & =h(x) u^{\beta(x)-1}+k(x, u) & & \text { in } \Omega ; \\
u & =0 & & \text { on } \partial \Omega .
\end{aligned}\right.
$$

Assume in addition either

(c1) $\frac{l}{h} \in L^{\infty}(\Omega)$

(c2) $k: \Omega \times \mathbb{R}^{+} \rightarrow \mathbb{R}^{+}$satisfying $\liminf _{t \rightarrow 0^{+}} \frac{k(x, t)}{t^{\alpha(x)-1}}>\|l\|_{L^{\infty}}$ uniformly in $x$.

Then $u$ is positive in $\Omega$.

Proof. We follow the idea of the proof of Theorem 1.1 in [19]. For the reader's convenience we have included the detailed proof. We rewrite our equation (3.1) under condition (c1) as follows:

$$
-\Delta_{p(x)} u+l(x) u^{\alpha(x)-1} \chi_{u \geq \lambda} \geq h(x) u^{\beta(x)-1}\left(1-\chi_{u \geq \lambda}\right)\left(1-\frac{l(x)}{h(x)} u^{\alpha(x)-\beta(x)}\right),
$$

since $\frac{l}{h} \in L^{\infty}(\Omega)$, we choose $\lambda \in(0,1)$ small enough such that for any $u(x) \leq \lambda$, we have $1-\frac{l(x)}{h(x)} u^{\alpha(x)-\beta(x)} \geq$ $1-\left\|\frac{l}{h}\right\|_{L^{\infty}(\Omega)} \lambda^{\alpha_{-}-\beta_{+}} \geq 0$.

Assuming condition (c2), we have

$$
-\Delta_{p(x)} u+l(x) u^{\alpha(x)-1} \chi_{u \geq \lambda} \geq k(x, u)-\left(1-\chi_{u \geq \lambda}\right) l(x) u^{\alpha(x)-1} .
$$

We choose $\lambda$ small enough such that for any $u(x) \leq \lambda$, we have $k(x, u)-l(x) u^{\alpha(x)-1} \geq 0$. Hence under both conditions, we get for any $x \in \Omega$,

$$
-\Delta_{p(x)} u+l(x) u^{\alpha(x)-1} \chi_{u \geq \lambda} \geq 0 .
$$

Suppose that there exists $x_{1}$ such that $u\left(x_{1}\right)=0$ then using the fact that $u$ is nontrivial, we can find $x_{2} \in \Omega$ and a ball $B\left(x_{2}, 2 C\right)$ in $\Omega$ such that $x_{1} \in \partial B\left(x_{2}, 2 C\right)$ and $u>0$ in $B\left(x_{2}, 2 C\right)$.

Let $a=\inf \left\{u(x):\left|x-x_{2}\right|=C\right\}$ then $a>0$ and choosing $x_{2}$ close enough to $x_{1}$ such that $0<a<\lambda$ and $\nabla u\left(x_{1}\right)=0$ since $u\left(x_{1}\right)=0$.

Denote the annulus $P=\left\{x \in \Omega: C<\left|x-x_{2}\right|<2 C\right\}$. We define $p_{1}=p\left(x_{1}\right), M=\sup \{|\nabla p(x)|: x \in P\}, \quad b=$ $8 M+2, l_{1}=-b \ln \left(\frac{a}{C}\right)+\frac{2(N-1)}{C}$ and

$$
j(t)=\frac{a}{e^{\frac{l_{1} C}{p_{1}-1}}-1}\left(e^{\frac{l_{1} t}{p_{1}-1}}-1\right) \forall t \in[0, C] .
$$

We have

$$
\frac{a}{C} e^{\frac{-l_{1} C}{p_{1}-1}}<j^{\prime}(0) \leq j^{\prime}(t) \leq j^{\prime}(C)<\frac{a}{C} e^{\frac{l_{1} C}{p_{1}-1}}
$$

and then

$$
\left(\frac{a}{C}\right)^{3} \leq j^{\prime}(t) \leq 1 \forall t \in[0, C] .
$$

We choose $C<1$ and using $\nabla u\left(x_{1}\right)=0, \frac{a}{C}<1$ small enough such that for any $x \in P$

$$
\frac{p(x)-1}{p_{1}-1} \geq \frac{1}{2}
$$

Without loss of generality we can take $x_{2}=0$ and we set $r=\left|x-x_{2}\right|=|x|, t=2 C-r$. For $t \in[0, C]$ and $r \in[C, 2 C]$, denote $w(r)=j(2 C-r)=j(t)$, then

$$
w^{\prime}(r)=-j^{\prime}(t), \quad w^{\prime \prime}(t)=j^{\prime \prime}(t) .
$$


From (3.2) and (3.3), we obtain

$$
\begin{aligned}
\operatorname{div}\left(|\nabla w|^{p(x)-2} \nabla w\right) & =(p(x)-1))\left(j^{\prime}(t)\right)^{p(x)-2} j^{\prime \prime}(t)-\frac{N-1}{r}\left(j^{\prime}(t)\right)^{p(x)-1}-\left(j^{\prime}(t)\right)^{p(x)-1} \ln \left(j^{\prime}(t)\right) \sum_{i=1}^{n} \frac{\partial p}{\partial x_{i}} \cdot \frac{x_{i}}{r} \\
& \geq\left(j^{\prime}(t)\right)^{p(x)-1}\left(\frac{1}{2} l_{1}+M \ln \left(j^{\prime}(t)\right)-\frac{N-1}{r}\right) \\
& \geq-\ln \left(\frac{a}{C}\right)\left(j^{\prime}(t)\right)^{p(x)-1} \geq 0 .
\end{aligned}
$$

Since $j(t)<a<\lambda$, we deduce

$$
-\operatorname{div}\left(|\nabla w|^{p(x)-2} \nabla w\right)+w^{\alpha(x)-1} \chi_{w \geq \lambda} \leq 0 .
$$

On $\partial P, w(C)=j(C)=a \leq u(x)$ and $w(2 C)=j(0)=0 \leq u(x)$. Then by Lemma 3.2, we obtain $w \leq u$ on $P$. Finally,

$$
\begin{aligned}
\lim _{s \rightarrow 0^{+}} \frac{u\left(x_{1}+s\left(x_{2}-x_{1}\right)\right)-u\left(x_{1}\right)}{s} & \geq \lim _{s \rightarrow 0^{+}} \frac{w\left(x_{1}+s\left(x_{2}-x_{1}\right)\right)-w\left(x_{1}\right)}{s} \\
& =j^{\prime}(0)>0
\end{aligned}
$$

which contradicts $\nabla u\left(x_{1}\right)=0$. Therefore, $u>0$ in $\Omega$.

Remark 3.1. Conditions (c1) and (c2) can be replaced by the condition that there exists $t_{0}$ such that $h(x) t^{\beta(x)-1}+$ $k(x, t)-l(x) t^{\alpha(x)-1} \geq 0$ for all $0<t<t_{0}$ and $x \in \Omega$.

Lemma 3.4. Under the same conditions of $h, l, k$ as in Lemma 3.3, let $u \in C^{1}(\bar{\Omega})$ be the nonnegative and nontrivial solution of (3.1), $x_{1} \in \partial \Omega, u\left(x_{1}\right)=0$ and $\Omega$ satisfies the interior ball condition at $x_{1}$, then $\frac{\partial u}{\partial \vec{n}}\left(x_{1}\right)<0$ where $\vec{n}$ is the outward unit normal vector at $x_{1}$.

Proof. Choose $C>0$ small enough such that $B\left(x_{2}, 2 C\right) \subset \Omega, x_{1} \in \partial B\left(x_{2}, 2 C\right)$. Then $x_{2}=x_{1}+2 C \vec{n}$, where $\vec{n}$ is the outward normal at $x_{1}$. Denote $P=\left\{x \in \Omega: C<\left|x-x_{2}\right|<2 C\right\}$ and by choosing $a$ such that $0<a<\lambda$, then by Lemma 3.3, there exist a subsolution $w \in C^{1}(\bar{P}) \cap C^{2}(P)$ of (3.1) in $P$ and $w$ satisfies $w \leq u$ in $P$ with $w\left(x_{1}\right)=0, \frac{\partial w}{\partial \vec{n}}\left(x_{1}\right)<0$. Hence, we get $\frac{\partial u}{\partial \vec{n}}\left(x_{1}\right) \leq \frac{\partial w}{\partial \vec{n}}\left(x_{1}\right)<0$.

\subsection{Proof of Theorem 1.3}

Proof of Theorem 1.3: We perform the proof along five steps. First we introduce notations. Define $F, G$ : $\Omega \times \mathbb{R} \rightarrow \mathbb{R}^{+}$as follows:

$$
F(x, t)= \begin{cases}\frac{h(x)}{q(x)} t^{q(x)} & \text { if } 0 \leq t<\infty ; \\ 0 & \text { if }-\infty<t<0,\end{cases}
$$

and

$$
G(x, t)= \begin{cases}\frac{l(x)}{s(x)} t^{s(x)} & \text { if } 0 \leq t<\infty ; \\ 0 & \text { if }-\infty<t<0 .\end{cases}
$$

We also extend the domain of $f$ and $g$ to all $\Omega \times \mathbb{R}$ by setting

$$
f(x, t)=\frac{\partial F}{\partial t}(x, t)=0 \text { and } g(x, t)=\frac{\partial G}{\partial t}(x, t)=0 \text { for }(x, t) \in \Omega \times(-\infty, 0) .
$$

Define the energy functional $\mathcal{E}: W_{0}^{1, p(x)}(\Omega) \cap L^{s(x)}(\Omega) \rightarrow \mathbb{R}$ by

$$
\varepsilon(u)=\int_{\Omega} \frac{|\nabla u|^{p(x)}}{p(x)} d x+\int_{\Omega} G(x, u(x)) d x-\int_{\Omega} F(x, u(x)) d x .
$$


Step 1 : Existence of a global minimizer

Since $W_{0}^{1, p(x)}(\Omega) \hookrightarrow L^{q(x)}(\Omega)$ (see Theorem 3.3.1 and Theorem 8.2.4 in [9]), the functional $\mathcal{E}$ is well-defined for every function $u \in W_{0}^{1, p(x)}(\Omega) \cap L^{s(x)}(\Omega)$.

For $\|u\|_{W_{0}^{1, p(x)}}$ large enough: by (1.3) or (1.4)

$$
\begin{aligned}
\mathcal{E}(u) \geq \int_{\Omega} \frac{|\nabla u|^{p(x)}}{p(x)}-\int_{\Omega} \frac{h(x)}{q(x)}|u|^{q(x)} & \geq \frac{1}{p_{-}}\|\nabla u\|_{L^{p(x)}}^{p_{-}}-C \rho_{q}(u) \\
& \geq \frac{1}{p_{-}}\|u\|_{W_{0}^{1, p(x)}}^{p_{-}}-C\|u\|_{W_{0}^{1, p(x)}}^{\tilde{q}}
\end{aligned}
$$

where $\tilde{q}=\left\{\begin{array}{ll}q_{-} & \text {if }\|u\|_{L^{p(x)}} \leq 1 \\ q_{+} & \text {if }\|u\|_{L^{p(x)}}>1\end{array}\right.$. Since $p_{-}>q_{+}$, this implies

$$
\mathcal{E}(u) \rightarrow \infty \text { as }\|u\|_{W_{0}^{1, p(x)}} \rightarrow+\infty .
$$

We argue similarly when $\|u\|_{L^{s(x)}} \rightarrow \infty$ and we deduce $\mathcal{E}$ is coercive. The continuity of $\mathcal{E}$ on $W_{0}^{1, p(x)}(\Omega) \cap L^{s(x)}(\Omega)$ is given by Theorem 3.2.8 and 3.2.9 of [9]. Hence we get the existence of at least one global minimizer, say $u_{0}$, to (3.4).

Step 2: Claim: $u_{0} \geq 0$ and $u_{0} \not \equiv 0$

Since $u_{0}$ is a global minimizer of $\mathcal{E}$ then $\mathcal{E}\left(u_{0}^{+}\right) \geq \mathcal{E}\left(u_{0}\right)$ where $u_{0}^{+}=\max \left\{u_{0}, 0\right\} \in W_{0}^{1, p(x)}(\Omega)$. Set $\Omega^{-}=\{x \in$ $\left.\Omega: u_{0}(x)<0\right\}$. We have

$$
\begin{aligned}
\mathcal{E}\left(u_{0}\right) & =\int_{\Omega} \frac{\left|\nabla u_{0}\right|^{p(x)}}{p(x)} d x+\int_{\Omega} G\left(x, u_{0}(x)\right) d x-\int_{\Omega} F\left(x, u_{0}(x)\right) d x \\
& =\mathcal{E}\left(u_{0}^{+}\right)+\int_{\Omega^{-}} \frac{\left|\nabla u_{0}\right|^{p(x)}}{p(x)} d x
\end{aligned}
$$

which implies $\int_{\Omega^{-}} \frac{\left|\nabla u_{0}\right|^{p(x)}}{p(x)}=0$ i.e. $\nabla u_{0}(x)=0$ a.e. in $\Omega^{-}$then by (1.3) and (1.4) we have $u_{0}=0$ a.e in $\Omega^{-}$. This implies that $u_{0} \geq 0$.

In order to show that $u_{0} \not \equiv 0$ in $\Omega$, we construct a function $v$ in $W_{0}^{1, p(x)}(\Omega) \cap L^{\infty}(\Omega)$ such that $\mathcal{E}(v)<0=\mathcal{E}(0)$. Precisely, consider $v=t \phi$ where $\phi \in C_{c}^{1}(\Omega), \phi \geq 0, \phi \not \equiv 0$ in $\Omega$ and for $0<t \leq 1$ small enough, we have

$$
\mathcal{E}(v) \leq t^{q_{+}}\left(c_{1} t^{p_{-}-q_{+}}+c_{2} t^{s_{-}-q_{+}}-c_{3}\right)
$$

where for any $i \in\{1,2,3\}, c_{i}$ are suitable constants independent of $t$. Hence, choosing $t$ small enough the right-hand side is negative and we conclude that $\mathcal{E}(t \phi)<0=\mathcal{E}(0)$ which implies $u_{0} \not \equiv 0$.

Step 3: $u_{0}$ satisfies the equation in (1.7)

Since $u_{0}$ is a global minimizer and $\mathcal{E}$ is $C^{1}$ on $W_{0}^{1, p(x)}(\Omega) \cap L^{s(x)}(\Omega)$, then for any $\phi \in W_{0}^{1, p(x)}(\Omega) \cap L^{s(x)}(\Omega)$, we have

$$
\left\langle\mathcal{E}^{\prime}\left(u_{0}\right), \phi\right\rangle=\int_{\Omega}\left|\nabla u_{0}\right|^{p(x)-2} \nabla u_{0} . \nabla \phi d x-\int_{\Omega} f\left(x, u_{0}\right) \phi d x+\int_{\Omega} g\left(x, u_{0}\right) \phi d x=0 .
$$

Step 4: Regularity and positivity of weak solutions

First we prove that all nonnegative weak solutions of (1.7) belongs to $L^{\infty}(\Omega)$ which yields $C^{1, \alpha}(\bar{\Omega})$ regularity. Let $K(x, t)=h(x) t^{q(x)-1}-l(x) t^{s(x)-1}$ and $\Lambda \stackrel{\text { def }}{=} \max \left\{\left\|\frac{h}{l}\right\|_{L^{\infty}}, 1\right\}^{1 /\left(s_{-}-q_{+}\right)}$

Then it is not difficult to show that for any $t \geq \Lambda, K(x, t) \leq 0$.

Let $u$ be a nonnegative function satisfying weakly the equation in (1.7). Then for any $\phi \in W_{0}^{1, p(x)}(\Omega) \cap$ $L^{s(x)}(\Omega)$,

$$
\int_{\Omega}|\nabla u|^{p(x)-2} \nabla u \cdot \nabla \phi d x=\int_{\Omega}\left(h(x) u^{q(x)-1}-l(x) u^{s(x)-1}\right) \phi(x) d x .
$$


Taking the testing function $\phi(x)=(u-\Lambda)^{+}$, we get

$$
\int_{\Omega}\left|\nabla(u-\Lambda)^{+}\right|^{p(x)} \leq 0 .
$$

By using (1.4), we deduce $\left\|(u-\Lambda)^{+}\right\|_{W_{0}^{1, p(x)}}=0$ which implies $u(x) \leq \Lambda$.

From Theorem 1.2 in [10], we get $u \in C^{1, \alpha}(\bar{\Omega})$ for some $\alpha \in(0,1)$.

Furthermore assuming $x \rightarrow \frac{l(x)}{h(x)}$ belongs to $L^{\infty}(\Omega)$, Lemma 3.3 yields $u>0$ in $\Omega$.

Step 5: Uniqueness of the positive solution of (1.7)

Let $u, v$ be two positive solutions of (1.7). Thus for any $\phi, \tilde{\phi} \in W_{0}^{1, p(x)}(\Omega) \cap L^{s(x)}(\Omega)$,

$$
\int_{\Omega}|\nabla u|^{p(x)-2} \nabla u \cdot \nabla \phi d x=\int_{\Omega}\left(h(x) u^{q(x)-1}-l(x) u^{s(x)-1}\right) \phi(x) d x
$$

and

$$
\int_{\Omega}|\nabla v|^{p(x)-2} \nabla v \cdot \nabla \tilde{\phi} d x=\int_{\Omega}\left(h(x) v^{q(x)-1}-l(x) v^{s(x)-1}\right) \tilde{\phi}(x) d x .
$$

By the previous steps, $u$ and $v$ belong to $C^{1}(\bar{\Omega})$ and Lemma 3.4 implies $u, v \in C_{d}^{0}(\bar{\Omega})^{+}$. Hence taking the testing functions as $\phi=\frac{\left(u^{p_{-}}-v^{p_{-}}\right)^{+}}{u^{p_{-}-1}}$ and $\tilde{\phi}=\frac{\left(v^{p_{-}}-u^{p_{-}}\right)^{-}}{v^{p_{-}-1}} \in W_{0}^{1, p(x)}(\Omega)$ (with the following notation $t^{-} \stackrel{\text { def }}{=}$ $\max \{0,-t\})$ and from Lemma 3.1 we obtain

$$
\begin{aligned}
0 \leq \int_{\{u>v\}}\left(|\nabla u|^{p(x)-2} \nabla u\right. & \left.-|\nabla v|^{p(x)-2} \nabla v\right) \cdot \nabla(u-v) d x \\
& =\int_{\{u>v\}} h(x)\left(u^{q(x)-p_{-}}-v^{q(x)-p_{-}}\right)\left(u^{p_{-}}-v^{p_{-}}\right) d x+\int_{\{u>v\}} l(x)\left(v^{s(x)-p_{-}}-u^{s(x)-p_{-}}\right)\left(u^{p_{-}}-v^{p_{-}}\right) d x .
\end{aligned}
$$

Since $q_{+} \leq p_{-} \leq s_{-}$, the both terms in right-hand side are nonpositive. This implies $v(x) \geq u(x)$ a.e in $\Omega$.

Finally reversing the role of $u$ and $v$, we get $u=v$.

Remark 3.2. Theorem 1.3 still holds when the condition $\frac{l}{h} \in L^{\infty}(\Omega)$ is replaced by $p_{+}<s_{-}$and using strong maximum principle in [19].

\section{Application to Fast diffusion equations}

In this section, we establish Theorems 1.4 and 1.5. To this aim, we use a time semi-discretization method associated to (1.9). With the help of accurate energy estimates about the related quasilinear elliptic equation and passing to the limit as the discretization parameter goes to 0 , we prove the existence and the properties of weak solutions to (1.8). In the subsection below, we study the associated elliptic problem.

\subsection{Study of the quasilinear elliptic problem associated to F.D.E.}

Consider the following problem

$$
\left\{\begin{aligned}
v^{2 q-1}-\lambda \Delta_{p(x)} v & =h_{0}(x) v^{q-1}+\lambda f(x, v) & & \text { in } \Omega ; \\
v & >0 & & \text { in } \Omega ; \\
v & =0 & & \text { on } \partial \Omega .
\end{aligned}\right.
$$

Assume $h_{0} \in L^{\infty}(\Omega)^{+}$and $f$ satisfies $\left(f_{1}\right)-\left(f_{3}\right)$. Then from $\left(f_{3}\right)$, we have $\left(f_{0}\right): \lim _{s \rightarrow+\infty} \frac{f(x, s)}{s^{p_{-}-1}}=0$ uniformly in $x \in \Omega$. Therefore, for any $\epsilon>0$, there exists a positive constant $C_{\epsilon}$ such that for any $(x, s) \in \Omega \times \mathbb{R}^{+}$:

$$
0 \leq f(x, s) \leq C_{\epsilon}+\epsilon s^{p_{-}-1} .
$$


We have the following preliminary result about (4.1):

Theorem 4.1. Let $\lambda>0, q \in\left(1, p_{-}\right], f: \Omega \times \mathbb{R}^{+} \rightarrow \mathbb{R}^{+}$satisfying $\left(f_{0}\right)$ and $\left(f_{1}\right)$ and $h_{0} \in L^{\infty}(\Omega)^{+}$. Then there exists a weak solution $v \in C^{1}(\bar{\Omega})$ to (4.1), i.e. for any $\phi \in \mathbf{W} \stackrel{\text { def }}{=} W_{0}^{1, p(x)}(\Omega) \cap L^{2 q}(\Omega)$

$$
\int_{\Omega} v^{2 q-1} \phi d x+\lambda \int_{\Omega}|\nabla v|^{p(x)-2} \nabla v \cdot \nabla \phi d x=\int_{\Omega} h_{0} v^{q-1} \phi d x+\lambda \int_{\Omega} f(x, v) \phi d x .
$$

In addition, if $\left(f_{2}\right)$ and $\left(f_{3}\right)$ hold then $v \in C_{d}^{0}(\bar{\Omega})^{+}$. Moreover if $v_{1}, v_{2} \in C_{d}^{0}(\bar{\Omega})^{+}$are two weak solutions to (4.1) corresponding to $h_{0}=h_{1}, h_{2} \in L^{\infty}(\Omega)^{+}$respectively, then we have

$$
\left\|\left(v_{1}^{q}-v_{2}^{q}\right)^{+}\right\|_{L^{2}} \leq\left\|\left(h_{1}-h_{2}\right)^{+}\right\|_{L^{2}} .
$$

Remark 4.1. (4.4) implies the uniqueness of the weak solution to (4.1) in $C_{d}^{0}(\bar{\Omega})^{+}$.

Proof. We perform the proof into several steps.

Step 1: Existence of a weak solution

Consider the energy functional $\mathcal{\partial}$ defined on $\mathbf{W}$ equipped with $\|\cdot\|_{\mathbf{W}}=\|\cdot\|_{W_{0}^{1, p(x)}}+\|\cdot\|_{L^{2 q}}$

$$
J(v)=\frac{1}{2 q} \int_{\Omega} v^{2 q} d x+\lambda \int_{\Omega} \frac{|\nabla v|^{p(x)}}{p(x)} d x-\frac{1}{q} \int_{\Omega} h_{0} D(v) d x-\lambda \int_{\Omega} F(x, v) d x
$$

where

$$
D(t)=\left\{\begin{array}{ll}
t^{q} & \text { if } 0 \leq t<\infty ; \\
0 & \text { if }-\infty<t<0,
\end{array} \text { and } F(x, t)= \begin{cases}\int_{0}^{t} f(x, s) d s & \text { if } 0 \leq t<\infty \\
0 & \text { if }-\infty<t<0\end{cases}\right.
$$

We also extend the domain of $f$ to all of $\Omega \times \mathbb{R}$ by setting $f(x, t)=\frac{\partial F}{\partial t}(x, t)=0$ for $(x, t) \in \Omega \times(-\infty, 0)$. From (4.2), Hölder inequality (1.5) and since $W_{0}^{1, p(x)} \hookrightarrow L^{p_{-}}(\Omega)$, we obtain

$$
\begin{aligned}
\mathcal{J}(v) & \geq \frac{1}{2 q}\|v\|_{L^{2 q}}^{2 q}+\lambda\|v\|_{W_{0}^{1, p(x)}}^{p_{-}}-\frac{1}{q}\left\|h_{0}\right\|_{L^{2}}\|v\|_{L^{2 q}}^{q}-\lambda C_{\epsilon} \int_{\Omega}|v| d x-\lambda \frac{\epsilon}{p_{-}} \int_{\Omega}|v|^{p_{-}} d x \\
& \geq \frac{1}{q}\|v\|_{L^{2 q}}^{q}\left(\frac{1}{2}\|v\|_{L^{2 q}}^{q}-\left\|h_{0}\right\|_{L^{2}}\right)+\lambda\|v\|_{W_{0}^{1, p(x)}}\left((1-\epsilon)\|v\|_{W_{0}^{1, p(x)}}^{p_{-}-1}-\tilde{C}\right) .
\end{aligned}
$$

Then by choosing $\epsilon$ small enough we conclude the coercivity of $\mathcal{\partial}$ on $\mathbf{W}$ and $\mathcal{\partial}$ is also continuous on $\mathbf{W}$ therefore we deduce the existence of a global minimizer $v_{0}$ to $\mathcal{J}$.

Furthermore we note

$$
\mathcal{J}\left(v_{0}\right) \geq \mathcal{J}\left(v_{0}^{+}\right)+\frac{1}{2 q} \int_{\Omega}\left(v_{0}^{-}\right)^{2 q} d x+\lambda \int_{\Omega} \frac{\left|\nabla v_{0}^{-}\right|^{p(x)}}{p(x)} d x
$$

which implies $v_{0} \geq 0$.

Now we claim that $v_{0} \not \equiv 0$ in $\Omega$. Since $\mathcal{J}(0)=0$, it is sufficient to prove the existence of $\tilde{v} \in \mathbf{W}$ such that $\mathcal{J}(\tilde{v})<0$. For that take $\tilde{v}=t \phi$ where $\phi \in C_{c}^{1}(\Omega)$ is nonnegative function such that $\phi \not \equiv 0$ and $t>0$ small enough.

Since $v_{0}$ is a global minimizer for the differentiable functional $\mathcal{J}$, we have that $v_{0}$ satisfies (4.3) i.e. $v_{0}$ is a weak solution to (4.1). From Corollary A.1 we infer that $v_{0} \in L^{\infty}(\Omega)$. Then by using Theorem A.2, we obtain, $v_{0} \in C^{1, \alpha}(\bar{\Omega})$ for some $\alpha \in(0,1)$.

From $\left(f_{2}\right)$ and Lemma 3.3 (with condition (c2)), we obtain $v_{0}>0$ and by Lemma 3.4 we get $\frac{\partial v_{0}}{\partial \vec{n}}<0$ on $\partial \Omega$. Therefore, $v_{0}$ belongs to $C_{d}^{0}(\bar{\Omega})^{+}$. 
Step 2: Contraction property (4.4)

Let $v_{1}$ and $v_{2}$ two positive weak solutions of (4.1) such that $v_{1}, v_{2} \in C_{d}^{0}(\bar{\Omega})^{+}$. For any $\phi, \Psi \in \mathbf{W}$ :

$$
\int_{\Omega} v_{1}^{2 q-1} \phi d x+\lambda \int_{\Omega}\left|\nabla v_{1}\right|^{p(x)-2} \nabla v_{1} \cdot \nabla \phi d x=\int_{\Omega} h_{1} v_{1}^{q-1} \phi d x+\lambda \int_{\Omega} f\left(x, v_{1}\right) \phi d x
$$

and

$$
\int_{\Omega} v_{2}^{2 q-1} \Psi d x+\lambda \int_{\Omega}\left|\nabla v_{2}\right|^{p(x)-2} \nabla v_{2} \cdot \nabla \Psi d x=\int_{\Omega} h_{2} v_{2}^{q-1} \Psi d x+\lambda \int_{\Omega} f\left(x, v_{2}\right) \Psi d x .
$$

Since $v_{1}, v_{2} \in C_{d}^{0}(\bar{\Omega})^{+}, \phi=\left(v_{1}-\frac{v_{2}^{q}}{v_{1}^{q-1}}\right)^{+}$and $\Psi=\left(v_{2}-\frac{v_{1}^{q}}{v_{2}^{q-1}}\right)^{-}$are well-defined and belong to $\mathbf{W}$. Subtracting the two above expressions and using $\left(f_{3}\right)$ together with Lemma 3.1 we obtain

$$
\int_{\Omega}\left(\left(v_{1}^{q}-v_{2}^{q}\right)^{+}\right)^{2} d x \leq \int_{\Omega}\left(h_{1}-h_{2}\right)\left(v_{1}^{q}-v_{2}^{q}\right)^{+} d x .
$$

Finally, applying the Hölder inequality we get (4.4).

From Theorem 4.1, we deduce the accretivity of $\mathcal{R}$ :

Corollary 4.1. Let $\lambda>0, q \in\left(1, p_{-}\right], f: \Omega \times \mathbb{R}^{+} \rightarrow \mathbb{R}^{+}$satisfying $\left(f_{1}\right)-\left(f_{3}\right)$ and $h_{0} \in L^{\infty}(\Omega)^{+}$. Consider the following problem

$$
\left\{\begin{array}{rlrl}
u+\lambda \mathcal{R} u & =h_{0}(x) & & \text { in } \Omega \\
u>0 & & \text { in } \Omega \\
u & =0 & & \text { on } \partial \Omega .
\end{array}\right.
$$

Then there exists a unique distributional solution $u \in \mathcal{D}(\mathcal{R}) \cap C^{1}(\bar{\Omega})$ of (4.6) i.e. $\forall \phi \in C_{c}^{1}(\Omega)$

$$
\int_{\Omega} u_{0} \phi d x+\lambda \int_{\Omega}\left|\nabla u_{0}^{1 / q}\right|^{p(x)-2} \nabla u_{0}^{1 / q} \cdot \nabla\left(\frac{\phi}{u_{0}^{(q-1) / q}}\right) d x=\int_{\Omega} h_{0} \phi d x+\lambda \int_{\Omega} \frac{f\left(x, u_{0}^{1 / q}\right)}{u_{0}^{(q-1) / q}} \phi d x .
$$

Moreover, if $u_{1}$ and $u_{2}$ are two distributional solutions of (4.6) in $\mathcal{D}(\mathcal{R}) \cap C^{1}(\bar{\Omega})$ associated to $h_{1}$ and $h_{2}$ respectively, then the operator $\mathcal{R}$ satisfies

$$
\left\|\left(u_{1}-u_{2}\right)^{+}\right\|_{L^{2}} \leq\left\|\left(u_{1}-u_{2}+\lambda\left(\mathcal{R} u_{1}-\mathcal{R} u_{2}\right)\right)^{+}\right\|_{L^{2}} .
$$

Proof. Define the energy functional $\mathcal{E}$ on $\dot{V}_{+}^{q} \cap L^{2}(\Omega)$ as $\mathcal{E}(u)=\mathcal{J}\left(u^{1 / q}\right)$ where $\mathcal{J}$ is defined in (4.5).

Let $\phi \in C_{c}^{1}(\Omega)$ and $v_{0}$ is the global minimizer of (4.5) which is also the weak solution of (4.1) and $u_{0}=v_{0}^{q}$ then there exists $t_{0}=t_{0}(\phi)>0$ such that for $t \in\left(-t_{0}, t_{0}\right), u_{0}+t \phi>0$. Hence we have

$$
\begin{aligned}
0 \leq \varepsilon\left(u_{0}+t \phi\right)-\varepsilon\left(u_{0}\right) & =\frac{1}{2 q}\left(\int_{\Omega}(t \phi)^{2} d x+\int_{\Omega} 2 t u_{0} \phi d x\right)-\frac{1}{q} \int_{\Omega} h t \phi d x \\
& +\lambda\left(\int_{\Omega} \frac{\left|\nabla\left(u_{0}+t \phi\right)^{1 / q}\right|^{p(x)}}{p(x)} d x-\int_{\Omega} \frac{\mid \nabla u_{0}^{1 / q} p^{p(x)}}{p(x)} d x\right) \\
& -\lambda\left(\int_{\Omega} F\left(x,\left(u_{0}+t \phi\right)^{1 / q}\right) d x-\int_{\Omega} F\left(x, u_{0}^{1 / q}\right) d x\right) .
\end{aligned}
$$

Then divide by $t$ and passing to the limits $t \rightarrow 0$ we obtain $u_{0}=v_{0}^{q}$ is the distributional solution of (4.6). Finally (4.7) and uniqueness follow from (4.4).

We now generalize some above results for a larger class of potentials $h_{0}$ : 


\subsection{Further results for (4.1) and uniqueness}

Theorem 4.2. Let $\lambda>0, f: \Omega \times \mathbb{R}^{+} \rightarrow \mathbb{R}^{+}$satisfying $\left(f_{1}\right)-\left(f_{3}\right)$ and $h_{0} \in L^{2}(\Omega)^{+}$and $q \in\left(1, p_{-}\right]$. Then there exists a positive weak solution $v \in \mathbf{W}$ of (4.1) in the sense of (4.3). Moreover assuming that $h_{0}$ belongs to $L^{v}(\Omega)$ for some $v>\max \left\{1, \frac{N}{p_{-}}\right\}, v \in L^{\infty}(\Omega)$.

Proof. Let $h_{n} \in C_{c}^{1}(\Omega)$ such that $h_{n} \geq 0$ and $h_{n} \rightarrow h$ in $L^{2}(\Omega)$. Define $\left(v_{n}\right) \subset C^{1, \alpha}(\bar{\Omega}) \cap C_{d}^{0}(\bar{\Omega})^{+}$as for a fixed $n$, $v_{n}$ is the unique positive weak solution of (4.1) with $h_{0}=h_{n}$ i.e. $v_{n}$ satisfies: for $\phi \in \mathbf{W}$

$$
\int_{\Omega} v_{n}^{2 q-1} \phi d x+\lambda \int_{\Omega}\left|\nabla v_{n}\right|^{p(x)-2} \nabla v_{n} . \nabla \phi d x=\int_{\Omega} h_{n} v_{n}^{q-1} \phi d x+\lambda \int_{\Omega} f\left(x, v_{n}\right) \phi d x .
$$

Since $(a-b)^{2 q} \leq\left(a^{q}-b^{q}\right)^{2}$ for any $q \geq 1$, (4.4) implies for any $n, p \in \mathbb{N}^{*}$

$$
\left\|\left(v_{n}-v_{p}\right)^{+}\right\|_{L^{2 q}} \leq\left\|\left(h_{n}-h_{p}\right)^{+}\right\|_{L^{2}}^{q}
$$

thus we deduce that $\left(v_{n}\right)$ converges to $v \in L^{2 q}(\Omega)$.

We infer that the limit $v$ does not depend on the choice of the sequence $\left(h_{n}\right)$. Indeed, consider $\tilde{h}_{n} \neq h_{n}$ such that $\tilde{h}_{n} \rightarrow h_{0}$ in $L^{2}(\Omega)$ and $\tilde{v}_{n}$ the positive solution of (4.1) corresponding to $\tilde{h}_{n}$ which converges to $\tilde{v}$.

Then, for any $n \in \mathbb{N}$, (4.4) implies

$$
\left\|\left(v_{n}^{q}-\tilde{v}_{n}^{q}\right)^{+}\right\|_{L^{2}} \leq\left\|\left(h_{n}-\tilde{h}_{n}\right)^{+}\right\|_{L^{2}}
$$

and passing to the limit we get $\tilde{v} \geq v$ and then by reversing the role of $v$ and $\tilde{v}$ we obtain $v=\tilde{v}$.

So define, for any $n \in \mathbb{N}^{*}, h_{n}=\min \{h, n\}$. Thus $\left(v_{n}\right)$ is nondecreasing and for any $n \in \mathbb{N}^{*}, v_{n} \leq v$ a.e. in $\Omega$ which implies $v \geq v_{1}>0$ in $\Omega$.

We choose $\phi=v_{n}$ in (4.8). Applying the Hölder inequality and (4.2), we obtain

$$
\begin{aligned}
\lambda \int_{\Omega}\left|\nabla v_{n}\right|^{p(x)} d x & \leq\left\|h_{n}\right\|_{L^{2}}\left\|v_{n}\right\|_{L^{2 q}}^{q}+\lambda C_{\epsilon}\left\|v_{n}\right\|_{L^{1}}+\lambda \epsilon\left\|v_{n}\right\|_{L^{p_{-}}}^{p_{-}} \\
& \leq C+\lambda \epsilon\left\|v_{n}\right\|_{L^{p_{-}}}^{p_{-}} .
\end{aligned}
$$

Assume $\left\|\nabla v_{n}\right\|_{L^{p(x)}} \geq 1$. Since $W_{0}^{1, p(x)}(\Omega) \hookrightarrow L^{p_{-}}(\Omega)$ and by (1.3) we deduce for some positive constant $C>0$ :

$$
\lambda \int_{\Omega}\left|\nabla v_{n}\right|^{p(x)} d x \leq C+\lambda \epsilon C \int_{\Omega}\left|\nabla v_{n}\right|^{p(x)} d x
$$

Choosing $\epsilon$ small enough and gathering with the case $\left\|\nabla v_{n}\right\|_{L^{p(x)}} \leq 1$, we conclude $\left(v_{n}\right)$ is uniformly bounded in $W_{0}^{1, p(x)}(\Omega)$ and $L^{p_{-}}(\Omega)$. Hence $v_{n}$ converges weakly to $v$ in $W_{0}^{1, p(x)}(\Omega)$ and by monotonicity of $\left(v_{n}\right)$ strongly in $L^{p_{-}}(\Omega)$ and in $L^{2 q}(\Omega)$. Taking now $\phi=v_{n}-v$ in (4.8), from (4.2) with $\epsilon=1$ and by Hölder inequality

$$
\left|\int_{\Omega} f\left(x, v_{n}\right)\left(v_{n}-v\right) d x\right| \leq C\left\|v_{n}-v\right\|_{L^{2 q}}+\left\|v_{n}\right\|_{L^{p_{-}}}^{p_{-}-1}\left\|v_{n}-v\right\|_{L^{p_{-}}} \rightarrow 0
$$

and

$$
\int_{\Omega} h_{n} v_{n}^{q-1}\left(v_{n}-v\right) d x \rightarrow 0 \text { and } \int_{\Omega} v_{n}^{2 q-1}\left(v_{n}-v\right) d x \rightarrow 0 .
$$

Finally (4.8) becomes

$$
\int_{\Omega}\left|\nabla v_{n}\right|^{p(x)-2} \nabla v_{n} \cdot \nabla\left(v_{n}-v\right) d x \rightarrow 0 .
$$

Then, since $v_{n} \rightarrow v$ in $W_{0}^{1, p(x)}(\Omega)$

$$
\int_{\Omega}\left(\left|\nabla v_{n}\right|^{p(x)-2} \nabla v_{n}-|\nabla v|^{p(x)-2} \nabla v\right) . \nabla\left(v_{n}-v\right) d x \rightarrow 0 .
$$


Lemma A.2 and Remark A.3 of [17] give the strong convergence of $v_{n}$ to $v$ in $W_{0}^{1, p(x)}(\Omega)$.

Since $\left(v_{n}^{2 q-1}\right)$ and $\left(h_{n} v_{n}^{q-1}\right)$ are uniformly bounded in $L^{2 q /(2 q-1)}(\Omega)$ and by (4.2), $f\left(x, v_{n}\right)$ is uniformly bounded in $L^{2 q / q-1}(\Omega)$ and $f\left(x, v_{n}\right) \rightarrow f(x, v)$ a.e. in $\Omega$. Then by Lebesgue dominated convergence theorem we have (up to a subsequence), for $\phi \in \mathbf{W}$

$$
\int_{\Omega} v_{n}^{2 q-1} \phi d x \rightarrow \int_{\Omega} v^{2 q-1} \phi d x, \int_{\Omega} h_{n} v_{n}^{q-1} \phi d x \rightarrow \int_{\Omega} h v^{q-1} \phi d x
$$

and

$$
\int_{\Omega} f\left(x, v_{n}\right) \phi d x \rightarrow \int_{\Omega} f(x, v) \phi d x .
$$

Finally we pass to the limit in (4.8) and we obtain $v$ is a weak solution of (4.1). To conclude corollary A.1 implies $v \in L^{\infty}(\Omega)$.

Remark 4.2. Let $v_{1}, v_{2}$ are the weak solutions of (4.1) obtained by Theorem 4.2 corresponding to $h_{1}, h_{2} \in$ $L^{2}(\Omega)^{+}, h_{1} \not \equiv h_{2}$ respectively. Then

$$
\left\|\left(v_{1}^{q}-v_{2}^{q}\right)^{+}\right\|_{L^{2}} \leq\left\|\left(h_{1}-h_{2}\right)^{+}\right\|_{L^{2}} .
$$

Remark 4.3. As in Step 1 of the proof of Theorem 4.1, we can alternatively prove the existence of a weak solution by global minimization method.

Under the hypothesis of Theorem 4.2 and with the help of Picone identity, the following theorem gives the uniqueness of the solution to (4.1).

Theorem 4.3. Let $v, \tilde{v}$ be respectively a subsolution and supersolution to (4.1) for $h \in L^{p_{0}}(\Omega), p_{0} \geq 2, h \geq 0$ and $f$ satisfies $\left(f_{1}\right)$ and $\left(f_{3}\right)$. Then $v \leq \tilde{v}$.

Proof. We have for any nonnegative $\phi, \Psi \in \mathbf{W}$

$$
\int_{\Omega} v^{2 q-1} \phi d x+\lambda \int_{\Omega}|\nabla v|^{p(x)-2} \nabla v \cdot \nabla \phi d x \leq \int_{\Omega} h v^{q-1} \phi d x+\lambda \int_{\Omega} f(x, v) \phi d x
$$

and

$$
\int_{\Omega} \tilde{v}^{2 q-1} \Psi d x+\lambda \int_{\Omega}|\nabla \tilde{v}|^{p(x)-2} \nabla \tilde{v} . \nabla \Psi d x \geq \int_{\Omega} h \tilde{v}^{q-1} \Psi d x+\lambda \int_{\Omega} f(x, \tilde{v}) \Psi d x
$$

Subtracting the above inequalities with test functions $\phi=\left(\frac{(v+\epsilon)^{q}-(\tilde{v}+\epsilon)^{q}}{(v+\epsilon)^{q-1}}\right)^{+}$and $\Psi=\left(\frac{(\tilde{v}+\epsilon)^{q}-(v+\epsilon)^{q}}{(\tilde{v}+\epsilon)^{q-1}}\right)^{-} \in \mathbf{W}$ for $\epsilon \in(0,1)$, we obtain

$$
\begin{aligned}
\int_{\{v>\tilde{v}\}} & \left(\frac{v^{2 q-1}}{(v+\epsilon)^{q-1}}-\frac{\tilde{v}^{2 q-1}}{(\tilde{v}+\epsilon)^{q-1}}\right)\left((v+\epsilon)^{q}-(\tilde{v}+\epsilon)^{q}\right) d x \\
& +\lambda \int_{\{v>\tilde{v}\}}|\nabla(v+\epsilon)|^{p(x)-2} \nabla(v+\epsilon) \cdot \nabla\left(\frac{(v+\epsilon)^{q}-(\tilde{v}+\epsilon)^{q}}{(v+\epsilon)^{q-1}}\right) d x \\
& +\lambda \int_{\{v>\tilde{v}\}} \mid \nabla(\tilde{v}+\epsilon)^{p(x)-2} \nabla(\tilde{v}+\epsilon) \cdot \nabla\left(\frac{(\tilde{v}+\epsilon)^{q}-(v+\epsilon)^{q}}{(\tilde{v}+\epsilon)^{q-1}}\right) d x \\
& \leq \int_{\{v>\tilde{v}\}} h\left(\frac{v^{q-1}}{(v+\epsilon)^{q-1}}-\frac{\tilde{v}^{q-1}}{(\tilde{v}+\epsilon)^{q-1}}\right)\left((v+\epsilon)^{q}-(\tilde{v}+\epsilon)^{q}\right) d x \\
& +\lambda \int_{\{v>\tilde{v}\}}\left(\frac{f(x, v)}{(v+\epsilon)^{q-1}}-\frac{f(x, \tilde{v})}{(\tilde{v}+\epsilon)^{q-1}}\right)\left((v+\epsilon)^{q}-(\tilde{v}+\epsilon)^{q}\right) d x .
\end{aligned}
$$


Since $\frac{\tilde{v}}{\tilde{v}+\varepsilon} \leq \frac{v}{v+\epsilon}<1$ in $\{v>\tilde{v}\}$, then we obtain

$$
\begin{aligned}
\left(\frac{v^{2 q-1}}{(v+\epsilon)^{q-1}}-\frac{\tilde{v}^{2 q-1}}{(\tilde{v}+\epsilon)^{q-1}}\right)\left((v+\epsilon)^{q}-(\tilde{v}+\epsilon)^{q}\right) & =\left(v^{q}\left(\frac{v}{v+\epsilon}\right)^{q-1}-\tilde{v}^{q}\left(\frac{\tilde{v}}{\tilde{v}+\epsilon}\right)^{q-1}\right)\left((v+\epsilon)^{q}-(\tilde{v}+\epsilon)^{q}\right) \\
& \leq v^{q}\left((v+\epsilon)^{q}-(\tilde{v}+\epsilon)^{q}\right) \leq v^{q}(v+\epsilon)^{q} \leq v^{q}(v+1)^{q} .
\end{aligned}
$$

In the same fashion, we have

$$
0 \leq h\left(\frac{v^{q-1}}{(v+\epsilon)^{q-1}}-\frac{\tilde{v}^{q-1}}{(\tilde{v}+\epsilon)^{q-1}}\right)\left((v+\epsilon)^{q}-(\tilde{v}+\epsilon)^{q}\right) \leq h(v+\epsilon)^{q} \leq h(v+1)^{q} .
$$

Moreover, as $\epsilon \rightarrow 0$

$$
\left(\frac{v^{2 q-1}}{(v+\epsilon)^{q-1}}-\frac{\tilde{v}^{2 q-1}}{(\tilde{v}+\epsilon)^{q-1}}\right)\left((v+\epsilon)^{q}-(\tilde{v}+\epsilon)^{q}\right) \rightarrow\left(v^{q}-\tilde{v}^{q}\right)^{2}
$$

and

$$
h\left(\frac{v^{q-1}}{(v+\epsilon)^{q-1}}-\frac{\tilde{v}^{q-1}}{(\tilde{v}+\epsilon)^{q-1}}\right)\left((v+\epsilon)^{q}-(\tilde{v}+\epsilon)^{q}\right) \rightarrow 0
$$

a.e. in $\Omega$. Then by Lebesgue dominated convergence theorem we have

$$
\int_{\{v>\tilde{v}\}}\left(\frac{v^{2 q-1}}{(v+\epsilon)^{q-1}}-\frac{\tilde{v}^{2 q-1}}{(\tilde{v}+\epsilon)^{q-1}}\right)\left((v+\epsilon)^{q}-(\tilde{v}+\epsilon)^{q}\right) d x \rightarrow \int_{\{v>\tilde{v}\}}\left(v^{q}-\tilde{v}^{q}\right)^{2} d x
$$

and

$$
\int_{\{v>\tilde{v}\}} h\left(\frac{v^{q-1}}{(v+\epsilon)^{q-1}}-\frac{\tilde{v}^{q-1}}{(\tilde{v}+\epsilon)^{q-1}}\right)\left((v+\epsilon)^{q}-(\tilde{v}+\epsilon)^{q}\right) d x \rightarrow 0 .
$$

Then by using Fatou's Lemma and $\left(f_{1}\right)$, we have

$$
\begin{aligned}
& -\liminf _{\epsilon \rightarrow 0} \int_{\{v>\tilde{\}}\}} \frac{f(x, v)}{(v+\epsilon)^{q-1}}(\tilde{v}+\epsilon)^{q} d x \leq-\int_{\{v>\tilde{v}\}} \frac{f(x, v)}{v^{q-1}} \tilde{v}^{q} d x, \\
& -\liminf _{\epsilon \rightarrow 0} \int_{\{v>\tilde{v}\}} \frac{f(x, \tilde{v})}{(\tilde{v}+\epsilon)^{q-1}}(v+\epsilon)^{q} d x \leq-\int_{\{v>\tilde{v}\}} \frac{f(x, \tilde{v})}{\tilde{v} q-1} v^{q} d x
\end{aligned}
$$

and

$$
\begin{aligned}
& \int_{\{v>\tilde{v}\}} f(x, v)(v+\epsilon) d x \rightarrow \int_{\{v>\tilde{v}\}} f(x, v) v d x, \\
& \int_{\{v>\tilde{v}\}} f(x, \tilde{v})(\tilde{v}+\epsilon) d x \rightarrow \int_{\{v>\tilde{v}\}} f(x, \tilde{v}) \tilde{v} d x .
\end{aligned}
$$

By Lemma 3.1 we have,

$$
\begin{aligned}
& \int_{\{v>\tilde{v}\}}|\nabla(v+\epsilon)|^{p(x)-2} \nabla(v+\epsilon) \cdot \nabla\left(\frac{(v+\epsilon)^{q}-(\tilde{v}+\epsilon)^{q}}{(v+\epsilon)^{q-1}}\right) d x \\
& \quad+\int_{\{v>\tilde{v}\}}|\nabla(\tilde{v}+\epsilon)|^{p(x)-2} \nabla(\tilde{v}+\epsilon) \cdot \nabla\left(\frac{(\tilde{v}+\epsilon)^{q}-(v+\epsilon)^{q}}{(\tilde{v}+\epsilon)^{q-1}}\right) d x \\
& \quad \geq 0 .
\end{aligned}
$$

Then plugging (4.10)-(4.14) and taking $\limsup _{\epsilon \rightarrow 0}$ in (4.9), we get by $\left(f_{3}\right)$

$$
0 \leq \int_{\{v>\tilde{v}\}}\left(v^{q}-\tilde{v}^{q}\right)^{2} d x \leq \lambda \int_{\{v>\tilde{v}\}}\left(\frac{f(x, v)}{v^{q-1}}-\frac{f(x, \tilde{v})}{\tilde{v}^{q-1}}\right)\left(v^{q}-\tilde{v}^{q}\right) d x \leq 0 .
$$

It implies $\tilde{v} \geq v$. 
Corollary 4.2. Let $\lambda>0, f: \Omega \times \mathbb{R}^{+} \rightarrow \mathbb{R}^{+}$satisfying $\left(f_{1}\right)-\left(f_{3}\right)$ and $h_{0} \in L^{2}(\Omega)^{+} \cap L^{y}(\Omega)$ where $y>\max \left\{1, \frac{N}{p_{-}}\right\}$. Then there exists a unique positive distributional solution $u \in \mathcal{D}(\mathcal{R}) \cap L^{\infty}(\Omega)$ of (4.6) in the same sense as in Corollary 4.1.

Moreover if $u_{1}$ and $u_{2}$ are two positive distributional solutions of (4.6) for $h_{1}, h_{2} \in L^{2}(\Omega)^{+}$then $\mathcal{R}$ satisfies

$$
\left\|\left(u_{1}-u_{2}\right)^{+}\right\|_{L^{2}} \leq\left\|\left(u_{1}-u_{2}+\lambda\left(\mathcal{R} u_{1}-\mathcal{R} u_{2}\right)\right)^{+}\right\|_{L^{2}} .
$$

Proof. Define the functional energy $\mathcal{E}$ on $\dot{V}_{+}^{q} \cap L^{2}(\Omega)$ as $\mathcal{E}(u)=\mathcal{J}\left(u^{1 / q}\right)$ where $\mathcal{\partial}$ is given by (4.5).

By Theorem 4.2, Remark 4.3 and Theorem 4.3, $v_{0}$ is the unique positive solution of (4.1) and then unique global minimizer of $\mathcal{J}$. We take $u_{0}=v_{0}^{q}$ and proceed as the proof of Corollary 4.1 and we obtain $u_{0}=v_{0}^{q}$ is a distributional solution of (4.6). Finally Remark 4.2 gives (4.15).

\subsection{Existence of a weak solution to (1.8)}

In this section, in light of Remark 1.3, we consider the problem (1.9) and establish the existence of weak solution when $v_{0} \in C_{d}^{0}(\bar{\Omega})^{+} \cap W_{0}^{1, p(x)}(\Omega)$. Proof of Theorem 1.4: Let $n^{\star} \in \mathbb{N}^{\star}$ and set $\Delta_{t}=T / n^{\star}$. For $0 \leq n \leq n^{\star}$, we define $t_{n}=n \Delta_{t}$.

Step 1 : Approximation of $h$

For $n \in\left\{1,2, \ldots n^{\star}\right\}$, we define for $t \in\left[t_{n-1}, t_{n}\right)$ and $x \in \Omega$

$$
h_{\Delta_{t}}(t, x)=h^{n}(x) \stackrel{\text { def }}{=} \frac{1}{\Delta_{t}} \int_{t_{n-1}}^{t_{n}} h(s, x) d s .
$$

Then by Jensen inequality,

$$
\begin{aligned}
\left\|h_{\Delta_{t}}\right\|_{L^{2}\left(Q_{T}\right)}^{2} & =\Delta_{t} \sum_{n=1}^{N}\left\|h^{n}\right\|_{L^{2}(\Omega)}^{2}=\Delta_{t} \sum_{n=1}^{N}\left\|\frac{1}{\Delta_{t}} \int_{t_{n-1}}^{t_{n}} h(s, x) d s\right\|_{L^{2}(\Omega)}^{2} \\
& \leq \sum_{n=1}^{N} \int_{t_{n-1}}^{t_{n}}\|h(s, .)\|_{L^{2}(\Omega)}^{2} d s \leq\|h\|_{L^{2}\left(Q_{T}\right)}^{2} .
\end{aligned}
$$

Hence $h_{\Delta_{t}} \in L^{2}\left(Q_{T}\right)$ and $h^{n} \in L^{2}(\Omega)$ and let $\epsilon>0$, then there exists a function $h_{\epsilon} \in C_{0}^{1}\left(Q_{T}\right)$ such that $\left\|h-h_{\epsilon}\right\|_{L^{2}\left(Q_{T}\right)}<\frac{\epsilon}{3}$.

Hence,

$$
\left\|\left(h_{\epsilon}\right)_{\Delta_{t}}-h_{\Delta_{t}}\right\|_{L^{2}\left(Q_{T}\right)} \rightarrow 0 .
$$

Since $\left\|h_{\epsilon}-\left(h_{\epsilon}\right)_{\Delta_{t}}\right\|_{L^{2}\left(Q_{T}\right)} \rightarrow 0$ as $\Delta_{t} \rightarrow 0$ then for small enough $\Delta_{t}$ we have

$$
\left\|h_{\Delta_{t}}-h\right\|_{L^{2}\left(Q_{T}\right)} \leq\left\|\left(h_{\epsilon}\right)_{\Delta_{t}}-h_{\Delta_{t}}\right\|_{L^{2}\left(Q_{T}\right)}+\left\|h_{\epsilon}-\left(h_{\epsilon}\right)_{\Delta_{t}}\right\|_{L^{2}\left(Q_{T}\right)}+\left\|h-h_{\epsilon}\right\|_{L^{2}\left(Q_{T}\right)}<\epsilon .
$$

Hence $h_{\Delta_{t}} \rightarrow h$ in $L^{2}\left(Q_{T}\right)$.

Step 2: Time discretization of (1.9)

Define the following implicit Euler scheme and for $n \geq 1, v_{n}$ is the weak solution of

$$
\left\{\begin{aligned}
\left(\frac{v_{n}^{q}-v_{n-1}^{q}}{\Delta_{t}}\right) v_{n}^{q-1}-\Delta_{p(x)} v_{n} & =h^{n} v_{n}^{q-1}+f\left(x, v_{n}\right) & & \text { in } \Omega ; \\
v_{n} & >0 & & \text { in } \Omega ; \\
v_{n} & =0 & & \text { on } \partial \Omega .
\end{aligned}\right.
$$

Note that the sequence $\left(v_{n}\right)_{n=1,2, \ldots, n^{*}}$ is well-defined. Indeed for $n=1$ the existence and the uniqueness of $v_{1} \in C^{1, \alpha}(\bar{\Omega}) \cap C_{d}^{0}(\bar{\Omega})^{+}$follows from Theorems 4.1 and 4.3 with $h=\Delta_{t} h^{1}+v_{0}^{q} \in L^{\infty}(\Omega)^{+}$. Hence by induction 
we obtain in the same way the existence and the uniqueness of the solution $v_{n}$ for any $n=2,3, \ldots, n^{\star}$ where $v_{n} \in C^{1, \alpha}(\bar{\Omega}) \cap C_{d}^{0}(\bar{\Omega})^{+}$.

Step 3: Existence of a subsolution and supersolution

Now we construct a subsolution and a supersolution $\underline{w}$ and $\bar{w}$ of (4.16) such that for each $n \in$ $\left\{0,1,2, \ldots, n^{*}\right\}, v_{n}$ satisfies $0<\underline{w} \leq v_{n} \leq \bar{w}$.

Rewrite (4.16) as

$$
v_{n}^{2 q-1}-\Delta_{t} \Delta_{p(x)} v_{n}=\left(\Delta_{t} h^{n}+v_{n-1}^{q}\right) v_{n}^{q-1}+\Delta_{t} f\left(x, v_{n}\right) .
$$

Then following arguments in the proof of Theorems 4.1 and 4.3, from Theorem A.2 and from Lemma 3.4, for any $\mu>0$ there exists a unique weak solution, $w_{\mu} \in C^{1, \alpha}(\bar{\Omega}) \cap C_{d}^{0}(\bar{\Omega})^{+}$, to

$$
\left\{\begin{aligned}
-\Delta_{p(x)} w & \left.=\mu\left(h_{0} w^{q-1}+f(x, w)\right)\right) & & \text { in } \Omega ; \\
w & >0 & & \text { in } \Omega ; \\
w & =0 & & \text { on } \partial \Omega .
\end{aligned}\right.
$$

Let $\mu_{1}<\mu_{2}$ and $w_{\mu_{1}}, w_{\mu_{2}}$ be weak solutions of (4.18). Then,

$$
\begin{aligned}
& \int_{\Omega}\left|\nabla w_{\mu_{1}}\right|^{p(x)-2} \nabla w_{\mu_{1}} . \nabla \phi d x=\mu_{1} \int_{\Omega}\left(h_{0} w_{\mu_{1}}^{q-1}+f\left(x, w_{\mu_{1}}\right)\right) \phi d x, \\
& \int_{\Omega}\left|\nabla w_{\mu_{2}}\right|^{p(x)-2} \nabla w_{\mu_{1}} . \nabla \psi d x=\mu_{2} \int_{\Omega}\left(h_{0} w_{\mu_{2}}^{q-1}+f\left(x, w_{\mu_{2}}\right)\right) \psi d x .
\end{aligned}
$$

Subtracting the last two equations with $\phi=\frac{\left(w_{\mu_{1}}^{q}-w_{\mu_{2}}^{q}\right)^{+}}{w_{\mu_{1}}^{q-1}}$ and $\psi=\frac{\left(w_{\mu_{2}}^{q}-w_{\mu_{1}}^{q}\right)^{-}}{w_{\mu_{2}}^{q-1}} \in W_{0}^{1, p(x)}(\Omega)$ we obtain, by Lemma 3.1 and $\left(f_{3}\right), w_{\mu_{1}} \leq w_{\mu_{2}}$.

Then by using Theorems A.2 and A.3, we can choose $\mu$ small enough such that $\left\|w_{\mu}\right\|_{C^{1, \alpha}(\bar{\Omega})} \leq C_{\mu_{0}}$ for all $\mu \leq \mu_{0}$ and $\left\|w_{\mu}\right\|_{L^{\infty}(\Omega)} \rightarrow 0$ as $\mu \rightarrow 0$. Therefore $\left\{w_{\mu}: \mu \leq \mu_{0}\right\}$ is uniformly bounded and equicontinuous in $C^{1}(\bar{\Omega})$ and by Arzela Ascoli theorem $\left\|w_{\mu}\right\|_{C^{1}(\bar{\Omega})} \rightarrow 0$ as $\mu \rightarrow 0$ up to a subsequence. Then by mean value theorem we can choose $\mu$ small enough such that there exists $\underline{w} \in C^{1, \alpha}(\bar{\Omega}) \cap C_{d}^{0}(\bar{\Omega})^{+}$such that $0<\underline{w} \stackrel{\text { def }}{=} w_{\mu} \leq v_{0}$. Also $\underline{w}$ is the subsolution of (4.17) for $n=1$ i.e.

$$
\int_{\Omega} \underline{w}^{2 q-1} \phi d x+\Delta_{t} \int_{\Omega}|\nabla \underline{w}|^{p(x)-2} \nabla \underline{w} . \nabla \phi d x \leq \Delta_{t} \int_{\Omega}\left(h^{1} \underline{w}^{q-1}+f(x, \underline{w})\right) \phi d x+\int_{\Omega} v_{0}^{q} \underline{w}^{q-1} \phi d x
$$

for all $\phi \in W_{0}^{1, p(x)}(\Omega)$ and $\phi \geq 0$. We also recall $v_{1}$ satisfies

$$
\int_{\Omega} v_{1}^{2 q-1} \psi d x+\Delta_{t} \int_{\Omega}\left|\nabla v_{1}\right|^{p(x)-2} \nabla v_{1} \cdot \nabla \psi d x=\Delta_{t} \int_{\Omega}\left(h^{1} v_{1}^{q-1}+f\left(x, v_{1}\right)\right) \psi d x+\int_{\Omega} v_{0}^{q} v_{1}^{q-1} \psi d x
$$

for all $\psi \in W_{0}^{1, p(x)}(\Omega)$.

By Theorem 4.3, we obtain, $\underline{w} \leq v_{1}$ and then by induction a subsolution $\underline{w}$ such that $0<\underline{w} \leq v_{n}$ for all $n=0,1,2, \ldots, n^{\star}$.

Now we construct a supersolution. For that, we consider the following problem:

$$
\left\{\begin{aligned}
-\Delta_{p(x)} w & =\|h\|_{L^{\infty}} w^{q-1}+f(x, w)+K & & \text { in } \Omega ; \\
w & >0 & & \text { in } \Omega ; \\
w & =0 & & \text { on } \partial \Omega .
\end{aligned}\right.
$$

As above, there exists a unique weak solution to (4.19), $\bar{w}_{K} \in C^{1}(\bar{\Omega}) \cap C_{d}^{0}(\bar{\Omega})^{+}$. Let $w_{K}$ be the unique weak solution of

$$
\left\{\begin{aligned}
-\Delta_{p(x)} w_{K}=K & \text { in } \Omega ; \\
w_{K}=0 & \text { on } \partial \Omega
\end{aligned}\right.
$$


From Theorem A.3, $w_{K} \geq C K^{1 /\left(p_{+}-1+v\right)} \operatorname{dist}(x, \partial \Omega)$ where $v \in(0,1)$ and $\left\|w_{K}\right\|_{L^{\infty}(\Omega)} \rightarrow \infty$ as $K \rightarrow \infty$. Then by weak comparison principle we can choose $K$ large enough such that there exists such that $v_{0} \leq w_{K}<\bar{w}^{=} \stackrel{\text { def }}{=} \bar{w}_{K}$. We easily check that $\bar{w}$ is the supersolution of (4.17) for $n=1$ i.e.

$$
\left.\int_{\Omega} \bar{w}^{2 q-1} \phi d x+\Delta_{t} \int_{\Omega}|\nabla \bar{w}|^{p(x)-2} \nabla \bar{w}\right) \cdot \nabla \phi d x \geq \Delta_{t} \int_{\Omega}\left(h^{1} \bar{w}^{q-1}+K+f(x, \bar{w}) \phi d x+\int_{\Omega} v_{0}^{q} \bar{w}^{q-1} \phi d x\right.
$$

for all $\phi \in W_{0}^{1, p(x)}(\Omega)$ and $\phi \geq 0$. From Theorem 4.3, we get $\bar{w} \geq v_{1}$ and then by induction we have $\bar{w} \geq v_{n}$ for all $n \in\left\{1,2, \ldots n^{\star}\right\}$.

Step 4: Energy estimates

Define the function for $n=1, \ldots, n^{\star}$ and $t \in\left[t_{n-1}, t_{n}\right)$

$$
v_{\Delta_{t}}(t)=v_{n} \text { and } \tilde{v}_{\Delta_{t}}(t)=\frac{t-t_{n-1}}{\Delta_{t}}\left(v_{n}^{q}-v_{n-1}^{q}\right)+v_{n-1}^{q}
$$

which satisfies

$$
v_{\Delta_{t}}^{q-1} \frac{\partial \tilde{v}_{\Delta_{t}}}{\partial t}-\Delta_{p(x)} v_{\Delta_{t}}=f\left(x, v_{\Delta_{t}}\right)+h^{n} v_{\Delta_{t}}^{q-1}
$$

Multiplying the equation (4.16) by $\frac{v_{n}^{q}-v_{n-1}^{q}}{v_{n}^{q-1}}$ and summing from $n=1$ to $n^{\prime} \leq n^{\star}$, we get

$$
\begin{gathered}
\sum_{n=1}^{n^{\prime}} \int_{\Omega} \Delta_{t}\left(\frac{v_{n}^{q}-v_{n-1}^{q}}{\Delta_{t}}\right)^{2} d x+\sum_{n=1}^{n^{\prime}} \int_{\Omega}\left|\nabla v_{n}\right|^{p(x)-2} \nabla v_{n} \cdot \nabla\left(\frac{v_{n}^{q}-v_{n-1}^{q}}{v_{n}^{q-1}}\right) d x \\
=\sum_{n=1}^{n^{\prime}} \int_{\Omega} h^{n}\left(v_{n}^{q}-v_{n-1}^{q}\right) d x+\sum_{n=1}^{n^{\prime}} \int_{\Omega} \frac{f\left(x, v_{n}\right)}{v_{n}^{q-1}}\left(v_{n}^{q}-v_{n-1}^{q}\right) d x .
\end{gathered}
$$

Then from Young inequality we have,

$$
\begin{aligned}
\sum_{n=1}^{n^{\prime}} \int_{\Omega} \Delta_{t} & \left(\frac{v_{n}^{q}-v_{n-1}^{q}}{\Delta_{t}}\right)^{2} d x+\sum_{n=1}^{n^{\prime}} \int_{\Omega}\left|\nabla v_{n}\right|^{p(x)-2} \nabla v_{n} \cdot \nabla\left(\frac{v_{n}^{q}-v_{n-1}^{q}}{v_{n}^{q-1}}\right) d x \\
& \leq \sum_{n=1}^{n^{\prime}} \Delta_{t}\left\|h^{n}\right\|_{L^{2}}^{2}+\frac{1}{4} \sum_{n=1}^{n^{\prime}} \int_{\Omega} \Delta_{t}\left(\frac{v_{n}^{q}-v_{n-1}^{q}}{\Delta_{t}}\right)^{2} d x \\
& +\sum_{n=1}^{n^{\prime}} \Delta_{t}\left\|\frac{f\left(x, v_{n}\right)}{v_{n}^{q-1}}\right\|_{L^{2}}^{2}+\frac{1}{4} \sum_{n=1}^{n^{\prime}} \int_{\Omega} \Delta_{t}\left(\frac{v_{n}^{q}-v_{n-1}^{q}}{\Delta_{t}}\right)^{2} d x
\end{aligned}
$$

i.e.

$$
\frac{1}{2} \sum_{n=1}^{n^{\prime}} \int_{\Omega} \Delta_{t}\left(\frac{v_{n}^{q}-v_{n-1}^{q}}{\Delta_{t}}\right)^{2} d x+\sum_{n=1}^{n^{\prime}} \int_{\Omega}\left|\nabla v_{n}\right|^{p(x)-2} \nabla v_{n} \cdot \nabla\left(\frac{v_{n}^{q}-v_{n-1}^{q}}{v_{n}^{q-1}}\right) d x \leq \sum_{n=1}^{n^{\prime}} \Delta_{t}\left\|h^{n}\right\|_{L^{2}}^{2}+\sum_{n=1}^{n^{\prime}} \Delta_{t}\left\|\frac{f\left(x, v_{n}\right)}{v_{n}^{q-1}}\right\|_{L^{2}}^{2} .
$$

Using $\underline{w} \leq v_{n} \leq \bar{w}$, from (4.2) and $q \leq \frac{N}{2}+1$, we obtain

$$
\int_{\Omega}\left|\frac{f\left(x, v_{n}\right)}{v_{n}^{(q-1)}}\right|^{2} d x \leq C_{1} \int_{\Omega} \frac{1}{\operatorname{dist}^{2(q-1)}(x, \partial \Omega)}+\operatorname{dist}^{2\left(p_{-}-q\right)}(x, \partial \Omega) d x \leq C
$$

where $C$ is independent of $n$. Then by Step 1, we obtain

$$
\left(\frac{\partial \tilde{v}_{\Delta_{t}}}{\partial t}\right) \text { is bounded in } L^{2}\left(Q_{T}\right) \text { uniformly in } \Delta_{t} \text {. }
$$


Now from Lemma 3.1, we have

$$
\begin{aligned}
\left|\nabla v_{n}\right|^{p(x)-2} \nabla v_{n} \cdot \nabla\left(\frac{v_{n-1}^{q}}{v_{n}^{q-1}}\right) & \leq\left|\nabla v_{n-1}\right|^{q}\left|\nabla v_{n}\right|^{p(x)-q} \\
& \leq \frac{q}{p(x)}\left|\nabla v_{n-1}\right|^{p(x)}+\frac{(p(x)-q)}{p(x)}\left|\nabla v_{n}\right|^{p(x)} .
\end{aligned}
$$

Then we obtain for any $n^{\prime} \geq 1$

$$
\begin{aligned}
\sum_{n=1}^{n^{\prime}} \Delta_{t}\left\|h^{n}\right\|_{L^{2}}^{2}+\sum_{n=1}^{n^{\prime}} \Delta_{t}\left\|\frac{f\left(x, v_{n}\right)}{v_{n}^{q-1}}\right\|_{L^{2}}^{2} & \geq \sum_{n=1}^{n^{\prime}} \int_{\Omega}\left|\nabla v_{n}\right|^{p(x)-2} \nabla v_{n} \cdot \nabla\left(\frac{v_{n}^{q}-v_{n-1}^{q}}{v_{n}^{q-1}}\right) d x \\
& \geq \sum_{n=1}^{n^{\prime}}\left[\int_{\Omega}\left|\nabla v_{n}\right|^{p(x)} d x-\int_{\Omega} \frac{q}{p(x)}\left|\nabla v_{n-1}\right|^{p(x)} d x-\int_{\Omega} \frac{(p(x)-q)}{p(x)}\left|\nabla v_{n}\right|^{p(x)} d x\right] \\
& \geq q \int_{\Omega} \frac{\left|\nabla v_{n^{\prime}}\right|^{p(x)}}{p(x)} d x-q \int_{\Omega} \frac{\left|\nabla v_{0}\right|^{p(x)}}{p(x)} d x
\end{aligned}
$$

which implies that

$$
\left(v_{\Delta_{t}}\right) \text { is bounded in } L^{\infty}\left(0, T ; W_{0}^{1, p(x)}(\Omega)\right) \text { uniformly in } \Delta_{t} .
$$

Since

$$
\nabla\left(\tilde{v}_{\Delta_{t}}^{1 / q}\right)=\frac{1}{q} \zeta \nabla v_{n}\left(\zeta+(1-\zeta)\left(\frac{v_{n-1}}{v_{n}}\right)^{q}\right)^{(1-q) / q}+(1-\zeta) \nabla v_{n-1}\left((1-\zeta)+\zeta\left(\frac{v_{n}}{v_{n-1}}\right)^{q}\right)^{(1-q) / q}
$$

where $\zeta=\frac{t-t_{n-1}}{\Delta_{t}}$, then we conclude that

$$
\left(\tilde{v}_{\Delta_{t}}^{1 / q}\right) \text { is bounded in } L^{\infty}\left(0, T ; W_{0}^{1, p(x)}(\Omega)\right) \text { uniformly in } \Delta_{t} \text {. }
$$

Since $\left(\frac{v_{n}}{v_{n-1}}\right)$ is uniformly bounded in $L^{\infty}(\Omega), v_{\Delta_{t}} \stackrel{\star}{\rightarrow} v$ and $\tilde{v}_{\Delta_{t}}^{1 / q} \stackrel{\star}{\rightarrow} \tilde{v}$ in $L^{\infty}\left(0, T ; W_{0}^{1, p(x)}(\Omega)\right)$. Furthermore using (4.23), we have

$$
\sup _{t \in(0, T)}\left\|\tilde{v}_{\Delta_{t}}^{1 / q}-v_{\Delta_{t}}\right\|_{L^{2 q}(\Omega)}^{2 q} \leq \sup _{t \in(0, T)}\left\|\tilde{v}_{\Delta_{t}}-v_{\Delta_{t}}^{q}\right\|_{L^{2}(\Omega)}^{2} \leq \Delta_{t} \rightarrow 0 \text { as } \Delta_{t} \rightarrow 0 .
$$

It follows from (4.26) that $v=\tilde{v}$. By mean value theorem and (4.23), we get that $\left(\tilde{v}_{\Delta_{t}}\right)_{\Delta_{t}}$ is equicontinuous in $C\left(0, T ; L^{r}(\Omega)\right)$ for $1<r \leq 2$. Thus using $\underline{w}^{q} \leq \tilde{v}_{\Delta_{t}} \leq \bar{w}^{q}$ together with the interpolation inequality $\|\cdot\|_{r} \leq$ $\|\cdot\|_{\infty}^{\alpha}\|\cdot\|_{2}^{1-\alpha}$, with $\frac{1}{r}=\frac{\alpha}{\infty}+\frac{1-\alpha}{2}$, we obtain that $\left(\tilde{v}_{\Delta_{t}}\right)_{\Delta_{t}}$ and $\left(\tilde{v}_{\Delta_{t}}^{1 / q}\right)_{\Delta_{t}}$ is equicontinuous in $C\left(0, T ; L^{r}(\Omega)\right)$ for any $1<r<+\infty$. Again using interpolation inequality and Sobolev embedding, we get as $\Delta_{t} \rightarrow 0^{+}$and up to a subsequence that for all $r>1$

$$
\tilde{v}_{\Delta_{t}} \rightarrow v^{q} \text { in } C\left(0, T ; L^{r}(\Omega)\right) \text {, }
$$

and

$$
v_{\Delta_{t}} \rightarrow v \text { in } L^{\infty}\left(0, T ; L^{r}(\Omega)\right) .
$$

From (4.23) and (4.27), we obtain

$$
\frac{\partial \tilde{v}_{\Delta_{t}}}{\partial t} \rightarrow \frac{\partial v^{q}}{\partial t} \text { in } L^{2}\left(Q_{T}\right) .
$$


Step 5 : $v$ satisfies (1.10)

Multiplying (4.21) by $\left(v_{\Delta_{t}}-v\right)$ and integrating by parts, we get

$$
\begin{aligned}
\int_{0}^{T} \int_{\Omega} v_{\Delta_{t}}^{q-1} & \frac{\partial \tilde{v}_{\Delta_{t}}}{\partial t}\left(v_{\Delta_{t}}-v\right) d x d t+\int_{0}^{T} \int_{\Omega}\left|\nabla v_{\Delta_{t}}\right|^{p(x)-2} \nabla v_{\Delta_{t}} . \nabla\left(v_{\Delta_{t}}-v\right) d x d t \\
= & \int_{0}^{T} \int_{\Omega} f\left(x, v_{\Delta_{t}}\right)\left(v_{\Delta_{t}}-v\right) d x d t+\int_{0}^{T} \int_{\Omega} h^{n} v_{\Delta_{t}}^{q-1}\left(v_{\Delta_{t}}-v\right) d x d t .
\end{aligned}
$$

From (4.28) and (4.29), we have

$$
\left|\int_{0}^{T} \int_{\Omega} v_{\Delta_{t}}^{q-1} \frac{\partial \tilde{v}_{\Delta_{t}}}{\partial t}\left(v_{\Delta_{t}}-v\right) d x d t\right|+\left|\int_{0}^{T} \int_{\Omega} h^{n} v_{\Delta_{t}}^{q-1}\left(v_{\Delta_{t}}-v\right) d x d t\right|=o_{\Delta_{t}}(1)
$$

and from (4.24), (4.25), (4.28) and Lebesgue Dominated convergence theorem,

$$
\int_{0}^{T} \int_{\Omega} f\left(x, v_{\Delta_{t}}\right)\left(v_{\Delta_{t}}-v\right) d x=o_{\Delta_{t}}(1)
$$

Then we obtain

$$
\int_{0}^{T} \int_{\Omega}\left|\nabla v_{\Delta_{t}}\right|^{p(x)-2} \nabla v_{\Delta_{t}} \cdot \nabla\left(v_{\Delta_{t}}-v\right) d x \rightarrow 0 \text { as } \Delta_{t} \rightarrow 0^{+} .
$$

Then from [Step 4, Proof of Theorem 1.1, [12]] and from classical compactness argument we get

$$
\left|\nabla v_{\Delta_{t}}\right|^{p(x)-2} \nabla v_{\Delta_{t}} \rightarrow|\nabla v|^{p(x)-2} \nabla v \text { in }\left(L^{p(x) /(p(x)-1)}\left(Q_{T}\right)\right)^{N} .
$$

From (4.26) and (4.27) we have,

$$
\begin{aligned}
\left\|v_{\Delta_{t}}^{q-1}-v^{q-1}\right\|_{L^{2}\left(Q_{T}\right)} & \leq\left\|v_{\Delta_{t}}^{q-1}-v^{q-1}\right\|_{L^{\infty}\left(0, T ; L^{2}\right)} \\
& \leq\left\|v_{\Delta_{t}}^{q-1}-v^{q-1}\right\|_{L^{\infty}\left(0, T ; L^{\frac{2 q}{q-1}}\right)} \\
& \leq\left\|v_{\Delta_{t}}^{q}-v^{q}\right\|_{L^{\infty}\left(0, T ; L^{2}\right)} \\
& \leq\left\|v_{\Delta_{t}}^{q}-\tilde{v}_{\Delta_{t}}\right\|_{L^{\infty}\left(0, T ; L^{2}\right)}+\left\|\tilde{v}_{\Delta_{t}}-v^{q}\right\|_{L^{\infty}\left(0, T ; L^{2}\right)} \rightarrow 0
\end{aligned}
$$

as $\Delta_{t} \rightarrow 0$. By Hölder inequality we have for $\phi \in C_{c}^{\infty}\left(Q_{T}\right)$

$$
\begin{aligned}
\int_{0}^{T} \int_{\Omega}\left(v_{\Delta_{t}}^{q-1} \frac{\partial \tilde{v}_{\Delta_{t}}}{\partial t}-\frac{\partial v^{q}}{\partial t} v^{q-1}\right) \phi d x & =\int_{0}^{T} \int_{\Omega} v_{\Delta_{t}}^{q-1}\left(\frac{\partial \tilde{v}_{\Delta_{t}}}{\partial t}-\frac{\partial v^{q}}{\partial t}\right) \phi d x+\int_{0}^{T} \int_{\Omega} \frac{\partial v^{q}}{\partial t}\left(v_{\Delta_{t}}^{q-1}-v^{q-1}\right) \phi d x \\
& \leq\left\|v_{\Delta_{t}}^{q-1} \phi\right\|_{L^{2}\left(Q_{T}\right)}\left\|\left(\frac{\partial \tilde{v}_{\Delta_{t}}}{\partial t}-\frac{\partial v^{q}}{\partial t}\right)\right\|_{L^{2}\left(Q_{T}\right)}+\left\|v_{\Delta_{t}}^{q-1}-v^{q-1}\right\|_{L^{2}\left(Q_{T}\right)}\left\|\phi \frac{\partial \tilde{v}_{\Delta_{t}}}{\partial t}\right\|_{L^{2}\left(Q_{T}\right)}
\end{aligned}
$$

and

$$
\begin{aligned}
\int_{0}^{T} \int_{\Omega}\left(h^{n} v_{\Delta_{t}}^{q-1}-h v^{q-1}\right) \phi d x & =\int_{0}^{T} \int_{\Omega} h^{n}\left(v_{\Delta_{t}}^{q-1}-v^{q-1}\right) \phi d x+\int_{0}^{T} \int_{\Omega}\left(h^{n}-h\right) v^{q-1} \phi d x \\
& \leq\left\|h^{n} \phi\right\|_{L^{2}\left(Q_{T}\right.}\left\|v_{\Delta_{t}}^{q-1}-v^{q-1}\right\|_{L^{2}\left(Q_{T}\right)}+\left\|v^{q-1} \phi\right\|_{L^{2}\left(Q_{T}\right)}\left\|h^{n}-h\right\|_{L^{2}\left(Q_{T}\right)} .
\end{aligned}
$$

Then from (4.23), (4.28), (4.29), (4.31) and Step 1 we obtain

$$
\begin{aligned}
& \int_{0}^{T} \int_{\Omega}\left(v_{\Delta_{t}}^{q-1} \frac{\partial \tilde{v}_{\Delta_{t}}}{\partial t}-\frac{\partial v^{q}}{\partial t} v^{q-1}\right) \phi d x \rightarrow 0, \\
& \int_{0}^{T} \int_{\Omega}\left(h^{n} v_{\Delta_{t}}^{q-1}-h v^{q-1}\right) \phi d x \rightarrow 0 \text { as } \Delta_{t} \rightarrow 0 .
\end{aligned}
$$


From (4.28) we have $f\left(x, v_{\Delta_{t}}\right) \rightarrow f(x, v)$ pointwise and from (4.24) together with (4.25) we have $\int_{\Omega} f\left(x, v_{\Delta_{t}}\right) \phi d x$ is bounded uniformly in $\Delta_{t}$. Then by Lebesgue dominated convergence theorem we have

$$
\int_{0}^{T} \int_{\Omega}\left(f\left(x, v_{\Delta_{t}}\right)-f(x, v)\right) \phi d x \rightarrow 0 \text { as } \Delta_{t} \rightarrow 0 .
$$

Then finally gathering (4.30), (4.32) and (4.33), we conclude by passing to the limits in equation (4.21) that $v$ is weak solution of (1.9).

Remark 4.4. For $q>\frac{N}{2}+1$, if $f$ satisfies $\lim _{s \rightarrow 0^{+}} \frac{f(x, s)}{s^{\alpha}}=0$ where $\alpha>q-1-\frac{N}{2}$ then Theorem 1.4 holds. Since $\underline{w} \leq v_{n} \leq \bar{w}$ then (4.22) is in this case

$$
\int_{\Omega}\left|\frac{f\left(x, v_{n}\right)}{v_{n}^{q-1}}\right|^{2} d x \leq C_{1} \int_{\Omega} \frac{\bar{w}^{2 \alpha}}{\operatorname{dist}^{2(q-1)}(x, \partial \Omega)} d x+C_{2} \leq C
$$

where $C$ is independent of $n$.

Remark 4.5. All the results in Section 4.1, Section 2 and Theorem 1.4 hold if we replace the assumption $\left(f_{2}\right)$ by $h \geq c>0$.

Proof of Theorem 1.5: For a given function $g$, let $\|g\|_{2^{+}} \stackrel{\text { def }}{=}\left\|[g]^{+}\right\|_{L^{2}(\Omega)}$. For $z \in \mathcal{D}(\mathcal{R})$ and $r, k \in L^{\infty}\left(Q_{T}\right)^{+}$ satisfying assumptions in Theorem 1.5, set

$$
\phi(t, s)=\|r(t)-k(s)\|_{2^{+}} \quad \forall(t, s) \in[0, T] \times[0, T]
$$

for $t \in[-T, T]$

$$
b(t, r, k)=\left\|u_{0}^{q}-z\right\|_{2^{+}}+\left\|v_{0}^{q}-z\right\|_{2^{+}}+|t|\|\mathcal{R} z\|_{2^{+}}+\int_{0}^{t^{+}}\|r(\tau)\|_{2^{+}} d \tau+\int_{0}^{t^{-}}\|k(\tau)\|_{2^{+}} d \tau
$$

and

is a solution of

$$
\psi(t, s)=b(t-s, r, k)+ \begin{cases}\int_{0}^{s} \phi(t-s+\tau, \tau) d \tau & \text { if } 0 \leq s \leq t \leq T \\ \int_{0}^{t} \phi(\tau, s-t+\tau) d \tau & \text { if } 0 \leq t \leq s \leq T\end{cases}
$$

$$
\left\{\begin{aligned}
\frac{\partial \psi}{\partial t}(t, s)+\frac{\partial \psi}{\partial s}(t, s) & =\phi(t, s) & & \text { on }(t, s) \in[0, T] \times[0, T] ; \\
\psi(t, 0) & =b(t, r, k) & & \text { on } t \in[0, T] ; \\
\psi(0, s) & =b(-s, r, k) & & \text { on } s \in[0, T] .
\end{aligned}\right.
$$

Define the following iterative scheme, $u^{0}=u_{0}^{q}$ and for $n \geq 1, u^{n}$ is the solution of

$$
\left\{\begin{aligned}
\frac{u^{n}-u^{n-1}}{\Delta_{t}}+\mathcal{R} u^{n} & =h^{n} & & \text { in } \Omega ; \\
u^{n} & =0 & & \text { on } \partial \Omega .
\end{aligned}\right.
$$

Note that the sequence $\left\{u^{n}\right\}_{n=1,2, \ldots, N}$ is well defined. Indeed for $n=1$ the existence and the uniqueness of $u^{1} \in \mathcal{D}(\mathcal{R})$ follows from Corollary 4.1 with $h=\Delta_{t} h^{1}+u^{0} \in L^{\infty}(\Omega)^{+}$and $\lambda=\Delta_{t}$. Hence by induction we obtain in the same way the existence of the solution $u^{n}$ for any $n=2,3, \ldots, N$ where $u^{n} \in \mathcal{D}(\mathcal{R})$.

Moreover let denote by $\left(u_{\epsilon}^{n}\right)$ the solution of (4.35) with $\Delta_{t}=\epsilon, h=r, r^{n}=\frac{1}{\epsilon} \int_{(n-1) \epsilon}^{n \epsilon} r(\tau,). d \tau$ and $\left(u_{\eta}^{m}\right)$ the solution of (4.35) with $\Delta_{t}=\eta, h=k, k^{m}=\frac{1}{\eta} \int_{(m-1) \eta}^{m \eta} k(\tau,). d \tau$ respectively i.e we have

$$
\left\{\begin{array}{c}
\frac{u_{\epsilon}^{n}-u_{\epsilon}^{n-1}}{\epsilon}+\mathcal{R} u_{\epsilon}^{n}=r^{n} \\
\frac{u_{\eta}^{m}-u_{\eta}^{m-1}}{\eta}+\mathcal{R} u_{\eta}^{m}=k^{m}
\end{array}\right.
$$


For $(n, m) \in \mathbb{N}^{\star}$, multiplying the equation in (4.36) by $\frac{\epsilon \eta}{\epsilon+\eta}$ and then subtracting the two expressions we get,

$$
\frac{\eta}{\eta+\epsilon}\left(u_{\epsilon}^{n}-u_{\epsilon}^{n-1}\right)+\frac{\eta \epsilon}{\eta+\epsilon}\left(\mathcal{R} u_{\epsilon}^{n}-\mathcal{R} u_{\eta}^{m}\right)-\frac{\epsilon}{\eta+\epsilon}\left(u_{\eta}^{m}-u_{\eta}^{m-1}\right)=\frac{\eta \epsilon}{\eta+\epsilon}\left(r^{n}-k^{m}\right) .
$$

Then we infer that

$$
u_{\epsilon}^{n}-u_{\eta}^{m}+\frac{\epsilon \eta}{\epsilon+\eta}\left(\mathcal{R} u_{\epsilon}^{n}-\mathcal{R} u_{\eta}^{m}\right)=\frac{\epsilon \eta}{\epsilon+\eta}\left(r^{n}-k^{m}\right)+\frac{\eta}{\epsilon+\eta}\left(u_{\epsilon}^{n-1}-u_{\eta}^{m}\right)+\frac{\epsilon}{\epsilon+\eta}\left(u_{\epsilon}^{n}-u_{\eta}^{m-1}\right) .
$$

Let $\Phi_{n, m}^{\epsilon, \eta}=\left\|u_{\epsilon}^{n}-u_{\eta}^{m}\right\|_{2^{+}}$and since $\mathcal{R}$ satisfies (4.15) and setting $\lambda=\frac{\epsilon \eta}{\epsilon+\eta}$, we get

$$
\begin{aligned}
\Phi_{n, m}^{\epsilon, \eta} & =\left\|u_{\epsilon}^{n}-u_{\eta}^{m}\right\|_{2^{+}} \leq\left\|u_{\epsilon}^{n}-u_{\eta}^{m}+\frac{\epsilon \eta}{\epsilon+\eta}\left(\mathcal{R} u_{\epsilon}^{n}-\mathcal{R} u_{\eta}^{m}\right)\right\|_{2^{+}} \\
& \leq \frac{\epsilon \eta}{\epsilon+\eta}\left\|r^{n}-k^{m}\right\|_{2^{+}}+\frac{\eta}{\epsilon+\eta}\left\|u_{\epsilon}^{n-1}-u_{\eta}^{m}\right\|_{2^{+}}+\frac{\epsilon}{\epsilon+\eta}\left\|u_{\epsilon}^{n}-u_{\eta}^{m-1}\right\|_{2^{+}} .
\end{aligned}
$$

Then by elementary calculations, we get

$$
\Phi_{n, 0}^{\epsilon, \eta}=\left\|u_{\epsilon}^{n}-u_{\eta}\right\|_{2+} \leq b\left(t_{n}, r_{\epsilon}, k_{\eta}\right)
$$

and

$$
\Phi_{0, m}^{\epsilon, \eta} \leq b\left(-s_{m}, r_{\epsilon}, k_{\eta}\right) .
$$

Then by using above computations we get , $\Phi_{n, m}^{\epsilon, \eta} \leq \psi_{n, m}^{\epsilon, \eta}$ where $\psi_{n, m}^{\epsilon, \eta}$ satisfies

$$
\psi_{n, m}^{\epsilon \eta}=\frac{\epsilon \eta}{\epsilon+\eta}\left\|\left(r^{n}-k^{m}\right)\right\|_{2^{+}}+\frac{\eta}{\epsilon+\eta}\left\|\psi_{n-1, m}^{\epsilon, \eta}\right\|_{2^{+}}+\frac{\epsilon}{\epsilon+\eta}\left\|\psi_{n, m-1}^{\epsilon, \eta}\right\|_{2^{+}}
$$

and $\psi_{n, 0}^{\epsilon, \eta}=b\left(t_{n}, r_{\epsilon}, k_{\eta}\right)$ and $\psi_{0, m}^{\epsilon, \eta}=b\left(-s_{m}, r_{\epsilon}, k_{\eta}\right)$.

For $(t, s) \in\left(t_{n-1}, t_{n}\right) \times\left(s_{m-1}, s_{m}\right)$, set $\phi^{\epsilon, \eta}(t, s)=\left\|r_{\epsilon}(t)-k_{\eta}(s)\right\|_{2^{+}}$,

$$
\psi^{\epsilon, \eta}=\psi_{n, m}^{\epsilon, \eta}, b_{\epsilon, \eta}(t, r, k)=b\left(t_{n}, r_{\epsilon}, k_{\eta}\right), b_{\epsilon, \eta}(-s, r, k)=b\left(-s_{m}, r_{\epsilon}, k_{\eta}\right) .
$$

Then by elementary calculations $\psi^{\epsilon, \eta}$ satisfies the following discrete version of (4.34),

$$
\left\{\begin{aligned}
\frac{\psi^{\epsilon, \eta}(t, s)-\psi^{\epsilon, \eta}(t-\epsilon, s)}{\epsilon}+\frac{\psi^{\epsilon, \eta}(t, s)-\psi^{\epsilon, \eta}(t, s-\eta)}{\eta} & =\phi^{\epsilon, \eta}(t, s) ; \\
\psi \epsilon, \eta(t, 0) & =b_{\epsilon, \eta}(t, r, k) ; \\
\psi^{\epsilon, \eta}(0, s) & =b_{\epsilon, \eta}(s, r, k) .
\end{aligned}\right.
$$

Since $r_{\epsilon} \rightarrow r$ in $L^{2}\left(Q_{T}\right)$ then $b_{\epsilon, \eta}(., r, k) \rightarrow b(., r, k)$ in $L^{\infty}([0, T])$ and $\phi^{\epsilon, \eta} \rightarrow \phi$ in $L^{\infty}([0, T] \times[0, T])$ and we deduce that $\rho_{\epsilon, \eta}=\left\|\psi^{\epsilon, \eta}-\psi\right\|_{L^{\infty}([0, T] \times[0, T])} \rightarrow 0$ (for more details see for instance [[20], Chapter 4, Lemma 4.3, page 136] and [[20], Chapter 4, Proof of Theorem 4.1, page 138]). Therefore,

$$
\left\|u_{\epsilon}(t)-u_{\eta}(s)\right\|_{2^{+}}=\Phi^{\epsilon, \eta}(t, s) \leq \psi^{\epsilon, \eta}(t, s) \leq \psi(t, s)+\rho_{\epsilon, \eta} .
$$

Since $u_{\epsilon}(t)=v_{\epsilon}^{q}(t)$ and $u_{\eta}(t)=v_{\eta}^{q}(t)$, we obtain

$$
\left\|v_{\epsilon}^{q}(t)-v_{\eta}^{q}(s)\right\|_{2^{+}}=\Phi^{\epsilon, \eta}(t, s) \leq \psi^{\epsilon, \eta}(t, s) \leq \psi(t, s)+\rho_{\epsilon, \eta} .
$$

From Theorem 1.4, $v_{\epsilon}^{q}$ and $v_{\eta}^{q}$ satisfies $0<\underline{w}<v_{\epsilon}, v_{\eta}<\bar{w}$ where $\underline{w}, \bar{w}$ are subsolution and supersolution defined in (4.18) and (4.19) and $v_{\epsilon}^{q} \rightarrow v_{1}^{q}$ and $v_{\eta}^{q} \rightarrow v_{2}^{q}$ a.e. in $\Omega$ where $v_{1}$ and $v_{2}$ are weak solutions of (1.9) with initial data $u_{0}, v_{0}$ respectively. Since $v_{\epsilon}^{q} \rightarrow v_{1}^{q}$ and $v_{\eta}^{q} \rightarrow v_{2}^{q}$ in $L^{\infty}\left(0, T ; L^{2}(\Omega)\right)$ and passing to the limit in (4.37) as $\epsilon, \eta \rightarrow 0$ with $t=s$ we get

$$
\begin{aligned}
\left\|v_{1}^{q}(t)-v_{2}^{q}(t)\right\|_{2^{+}} & \leq\left\|v_{1}^{q}(t)-v_{\epsilon}^{q}(t)\right\|_{2^{+}}+\left\|v_{\eta}^{q}(t)-v_{2}^{q}(t)\right\|_{2^{+}}+\left\|v_{\epsilon}^{q}(t)-v_{\eta}^{q}(t)\right\|_{2^{+}} \\
& \leq\left\|u_{0}^{q}-z\right\|_{2^{+}}+\left\|v_{0}^{q}-z\right\|_{2^{+}}+\int_{0}^{t}\|r(y)-k(y)\|_{2^{+}} d y .
\end{aligned}
$$

Then (1.11) follows since we can choose $z$ arbitrary close to $v_{0}^{q}$ and with $r=h, k=g$. 


\section{An application to nonhomogeneous operators}

In this final section, we prove Theorem 1.6. To this aim, we first study the properties of a related energy functional. Let $m \geq 1$ and $K: \Omega \times \mathbb{R}^{N} \rightarrow \mathbb{R}^{+}$be a continuous differentiable function which satisfies the following conditions:

(k1) $K \in C^{1}\left(\Omega \times \mathbb{R}^{N}\right) \cap C^{2}\left(\Omega \times \mathbb{R}^{N} \backslash\{0\}\right)$.

(k2) Ellipticity condition: $\exists k_{1} \geq 0$ and $y \in(0, \infty)$ such that

$$
\sum_{i, j=1}^{N} \frac{\partial^{2} K}{\partial \xi_{i} \partial \xi_{j}}(x, \xi) \eta_{i} \eta_{j} \geq y\left(k_{1}+|\xi|\right)^{m-2}|\eta|^{2} .
$$

(k3) Growth condition: $\exists k_{2} \geq 0$ and $\Gamma \in(0, \infty)$ such that

$$
\sum_{i, j=1}^{N}\left|\frac{\partial^{2} K}{\partial \xi_{i} \partial \xi_{j}}(x, \xi)\right| \leq \Gamma\left(k_{2}+|\xi|\right)^{m-2}
$$

for all $\xi \in \mathbb{R}^{N} \backslash\{0\}$ and $\eta \in \mathbb{R}^{N}$.

Remark 5.1. From the assumption (k2), it follows that $K$ is strictly convex and from (k1)-(k3) there exists some positive constant $y_{1}$ and $y_{2}$ with $0<y_{1} \leq y_{2}<+\infty$ and some nonnegative constants $\Gamma_{1}$ and $\Gamma_{2}$ such that

$$
y_{1}|\xi|^{m}-\Gamma_{1} \leq K(x, \xi) \leq y_{2}|\xi|^{m}+\Gamma_{2}
$$

for $x \in \Omega$ and $\xi \in \mathbb{R}^{N} \backslash\{0\}$.

Consider the associated functional $\mathcal{J}_{m}$ defined by

$$
\partial_{m}(u) \stackrel{\text { def }}{=} \int_{\Omega} \frac{|u|^{p(x)}}{p(x)} K\left(x, \frac{\nabla u}{u}\right)^{\frac{p(x)}{m}} d x .
$$

for any positive function $u \in W_{0}^{1, p(x)}(\Omega)$. Now we extend Lemma 2.4 in [21] as follows:

Theorem 5.1. Let $K: \Omega \times \mathbb{R}^{N} \rightarrow \mathbb{R}^{+}$satisfying (k1)-(k3) for some $m \in\left[1, p_{-}\right]$. Then, the function $\mathcal{E}: \dot{V}_{+}^{m} \cap$ $L^{\infty}(\Omega) \rightarrow \mathbb{R}^{+}$, defined by $\mathcal{E}(u) \stackrel{\text { def }}{=} \partial_{m}\left(u^{1 / m}\right)$, is ray-strictly convex (even strictly convex if $p(\cdot) \not \equiv m$ ).

Proof. We observe that for $u \in \dot{V}_{+}^{m} \cap L^{\infty}(\Omega)$

$$
\varepsilon(u)=\int_{\Omega} \frac{1}{p(x)}\left(u K\left(x, \frac{\nabla u}{m u}\right)\right)^{\frac{p(x)}{m}} d x .
$$

Therefore, since for $1 \leq m \leq p_{-}, t \rightarrow t^{p(x) / m}$ is convex in $\mathbb{R}^{+}$(even strictly convex if $p(x)>m$ ) it is enough to prove that

$$
\dot{V}_{+}^{m} \ni u \rightarrow u K\left(x, \frac{\nabla u}{m u}\right)
$$


is ray-strictly convex. To achieve this goal, let $\theta \in(0,1)$ and $u_{1}, u_{2} \in \dot{V}_{+}^{m}$ then by using the strict convexity of $K$ we obtain, for $x \in \Omega$

$$
\begin{aligned}
& \left((1-\theta) u_{1}+\theta u_{2}\right) K\left(x, \frac{(1-\theta) \nabla u_{1}+\theta \nabla u_{2}}{m\left((1-\theta) u_{1}+\theta u_{2}\right)}\right) \\
& =\left((1-\theta) u_{1}+\theta u_{2}\right) K\left(x, \frac{(1-\theta) u_{1}}{\left((1-\theta) u_{1}+\theta u_{2}\right)} \frac{\nabla u_{1}}{m u_{1}}+\frac{\theta u_{2}}{\left((1-\theta) u_{1}+\theta u_{2}\right)} \frac{\nabla u_{2}}{m u_{2}}\right) \\
& \leq\left((1-\theta) u_{1}+\theta u_{2}\right)\left(\frac{(1-\theta) u_{1}}{\left((1-\theta) u_{1}+\theta u_{2}\right)} K\left(x, \frac{\nabla u_{1}}{m u_{1}}\right)\right. \\
& \left.\quad+\frac{\theta u_{2}}{\left((1-\theta) u_{1}+\theta u_{2}\right)} K\left(x, \frac{\nabla u_{2}}{m u_{2}}\right)\right) \\
& =(1-\theta) u_{1} K\left(x, \frac{\nabla u_{1}}{m u_{1}}\right)+\theta u_{2} K\left(x, \frac{\nabla u_{2}}{m u_{2}}\right) .
\end{aligned}
$$

The above inequality is always strict unless $\frac{\nabla u_{1}}{u_{1}}=\frac{\nabla u_{2}}{u_{2}}$, i.e. $u_{1} / u_{2} \equiv$ Const.

Proof of Theorem 1.6: Consider the functional $\mathcal{J}_{\epsilon}: W_{0}^{1, p(x)}(\Omega) \rightarrow \mathbb{R}$, defined by

$$
J_{\epsilon}(u)=\int_{\Omega} \frac{\left(|\nabla u|^{2}+\epsilon u^{2}\right)^{p(x) / 2}}{p(x)} d x-\int_{\Omega} G(x, u) d x
$$

where the potential $G(x, t)$ defined as

$$
G(x, t)= \begin{cases}\int_{0}^{t} g(x, s) d s & \text { if } 0 \leq t<\infty ; \\ 0 & \text { if }-\infty<t<0 .\end{cases}
$$

Assumptions $\left(f_{1}\right),(\tilde{g})$ and Remark 5.1 ensure that $\partial_{\epsilon}$ is well defined, coercive and continuous. Then there exists at least one global minimizer of $\mathcal{J}_{\epsilon}$ on $W_{0}^{1, p(x)}(\Omega)$, say $u_{0}$. We can easily prove that $u_{0}$ is nonnegative and nontrivial.

Since $\mathcal{J}_{\epsilon}$ is differentiable, we deduce that $u_{0}$ is a weak solution of (1.12). Now from Theorems A.1 and A.2 in Appendix A, we obtain that any weak solution $u$ to (1.12) belongs to $C^{1, \alpha}(\bar{\Omega})$ for some $\alpha \in(0,1)$ and $u>0$ in $\Omega$ and $\frac{\partial u}{\partial \vec{n}}<0$ on $\partial \Omega$. Therefore any weak solution belongs to $C_{d}^{0}(\bar{\Omega})^{+}$.

Now we prove that $u_{0}$ is the unique weak solution to (1.12). Let $W: \dot{V}_{+}^{m} \rightarrow \mathbb{R}$ defined by

$$
W(u)=\partial_{\epsilon}\left(u^{1 / m}\right)=\int_{\Omega} \frac{\left(\left|\nabla\left(u^{1 / m}\right)\right|^{2}+\epsilon\left(u^{1 / m}\right)^{2}\right)^{p(x) / 2}}{p(x)} d x-\int_{\Omega} G\left(x, u^{1 / m}\right) d x .
$$

The assumption ( $\tilde{g})$ together with Theorem 5.1 with $K(x, \xi)=\left(\epsilon+|\xi|^{2}\right)^{m / 2}$ imply that $W$ is strictly convex. Let $u_{1}$ a weak solution to (1.12). Then setting $v_{0} \stackrel{\text { def }}{=} u_{0}^{m}, v_{1} \stackrel{\text { def }}{=} u_{1}^{m} \in \dot{V}_{+}^{m}$ and $t \in[0,1]$, we define $\xi(t) \stackrel{\text { def }}{=}$ $J_{\epsilon}\left(\left((1-t) v_{0}+t v_{1}\right)^{1 / m}\right)$. Since $u_{0}$ and $u_{1}$ belong to $C_{d}^{0}(\bar{\Omega})^{+}$, $\xi$ is differentiable in $[0,1]$. From the convexity of $\mathcal{E}$, we have for any $t \in[0,1]$

$$
\xi^{\prime}(0) \leq \xi^{\prime}(t) \leq \xi^{\prime}(1) .
$$

Since $u_{0}$ and $u_{1}$ are weak solutions to (1.12), $\xi^{\prime}(0)=\xi^{\prime}(1)=0$ and from (5.1) we get that $\xi$ is constant which contradicts the strict convexity of $\mathcal{E}$ unless $u_{0} \equiv u_{1}$.

\section{A Appendix}

In this section, we recall the following regularity of weak solutions of quasilinear elliptic differential equation

$$
\left\{\begin{aligned}
\operatorname{div} A(x, u, D u)+B(x, u, D u) & =0 & & \text { on } \Omega ; \\
u & =0 & & \text { on } \partial \Omega .
\end{aligned}\right.
$$


Now we recall the boundedness and $C^{0, \alpha}(\bar{\Omega})$ regularity results of weak solutions of (A.1) satisfying the following growth conditions:

$$
\begin{aligned}
& A(x, u, z) z \geq a_{0}|z|^{p(x)}-b|u|^{r(x)}-c ; \\
& |A(x, u, z)| \leq a_{1}|z|^{p(x)-1}+b|u|^{\sigma(x)}+c ; \\
& |B(x, u, z)| \leq a_{2}|z|^{\alpha(x)}+b|u|^{r(x)-1}+c
\end{aligned}
$$

where $a_{0}, a_{1}, a_{2}, b, c$ are positive constants and $p^{*}$ is the Sobolev embedding exponent of $p$ and

$$
p(x) \leq r(x)<p^{*}(x), \sigma(x)=\frac{p(x)-1}{p(x)} r(x) \text { and } \alpha(x)=\frac{r(x)-1}{r(x)} p(x) .
$$

Theorem A.1. ([11], Theorem 4.1 and Theorem 4.4) Let (A.2)-(A.3) hold and $p \in \mathcal{P}^{\log }(\Omega)$. If $u \in W^{1, p(x)}(\Omega)$ is a weak solution of (A.1), then $u \in C^{0, \alpha}(\overline{\Omega)}$.

Theorem A.2 below ensures $C^{1, \alpha}(\bar{\Omega})$ regularity to weak solutions of (A.1) under the additional assumptions on $p, A$ and $B$ :

Assumptions $\left(\mathbf{A}_{\mathbf{k}}\right): A=\left(A_{1}, A_{2}, \ldots, A_{n}\right) \in C\left(\bar{\Omega} \times \mathbb{R} \times \mathbb{R}^{N}, \mathbb{R}^{N}\right)$. For every $(x, u) \in \bar{\Omega} \times \mathbb{R}, A(x, u,.) \in$ $C^{1}\left(\mathbb{R}^{N} \backslash\{0\}, \mathbb{R}^{N}\right)$, there exist a nonnegative constants $k_{1}, k_{2}, k_{3} \geq 0$, a nonincreasing continuous function $\lambda:[0, \infty) \rightarrow(0, \infty)$ and a nondecreasing continuous function $\Lambda:[0, \infty) \rightarrow(0, \infty)$ such that for all $x, x_{1}, x_{2} \in$ $\bar{\Omega}, u, u_{1}, u_{2} \in \mathbb{R}, \eta \in \mathbb{R}^{N} \backslash\{0\}$ and $\xi=\left(\xi_{1}, \xi_{2}, \ldots, \xi_{n}\right) \in \mathbb{R}^{N}$, the following conditions are satisfied

$$
\begin{aligned}
& A(x, u, 0)=0, \\
& \sum_{i, j} \frac{\partial A_{j}(x, u, \eta)}{\partial \eta_{i}}(x, u, \eta) \xi_{i} \xi_{j} \geq \lambda(|u|)\left(k_{1}+|\eta|^{2}\right)^{(p(x)-2) / 2}|\xi|^{2}, \\
& \sum_{i, j}\left|\frac{\partial A_{j}(x, u, \eta)}{\partial \eta_{i}}(x, u, \eta)\right| \leq \Lambda(|u|)\left(k_{2}+|\eta|^{2}\right)^{(p(x)-2) / 2} \text { and } \\
& \left|A\left(x_{1}, u_{1}, \eta\right)-A\left(x_{2}, u_{2}, \eta\right)\right| \leq \Lambda\left(\max \left\{\left|u_{1}\right|,\left|u_{2}\right|\right\}\right)\left(\left|x_{1}-x_{2}\right|^{\beta_{1}}+\left|u_{1}-u_{2}\right|^{\beta_{2}}\right) \\
& \quad \times\left[\left(k+|\eta|^{2}\right)^{\left(p\left(x_{1}\right)-2\right) / 2}+\left(k+|\eta|^{2}\right)^{\left(p\left(x_{2}\right)-2\right) / 2}\right]|\eta|\left(1+\left|\log \left(k_{3}+|\eta|^{2}\right)\right|\right) .
\end{aligned}
$$

Assumption (B) : $B: \bar{\Omega} \times \mathbb{R} \times \mathbb{R}^{N} \rightarrow \mathbb{R}$, the function $B(x, u, \eta)$ is measurable in $x$ and is continuous in $(u, \eta$, and

$$
|B(x, u, \eta)| \leq \Lambda(|u|)\left(1+|\eta|^{p(x)}\right), \quad \forall(x, u, \eta) \in \bar{\Omega} \times \mathbb{R} \times \mathbb{R}^{N} .
$$

Theorem A.2. ([10], Theorem 1.2) Let assumptions $\left(\mathbf{A}_{\mathbf{k}}\right),(\mathbf{B})$ hold. Assume $p$ belongs to $C^{0, \beta}(\bar{\Omega})$, for some $\beta \in$ $(0,1)$. Suppose that $\Omega$ satisfy $(\Omega)$. If $u \in W_{0}^{1, p(x)}(\Omega) \cap L^{\infty}(\Omega)$ is a weak solution of (A.1), then $u \in C^{1, \alpha}(\bar{\Omega})$ where $\alpha \in(0,1)$ and $\|u\|_{C^{1, \alpha}(\bar{\Omega})}$ depends upon $p_{-}, p_{+}, \lambda(M), \Lambda(M), \beta_{1}, \beta_{2}, M, \Omega$ where $M \stackrel{\text { def }}{=}\|u\|_{L^{\infty}(\Omega)}$.

In the next theorem, we recall some results contained in Lemma 2.1 of [22] and Lemma 3.2 of [12]. Set $\varrho=$ $\frac{p_{-}}{2|\Omega|^{1 / N} C_{0}}$ where $C_{0}$ is the best embedding constant of $W_{0}^{1,1}(\Omega) \subset L^{\frac{N}{N-1}}(\Omega)$.

Theorem A.3. Let $K>0$ and $w_{K} \in W_{0}^{1, p(x)}(\Omega) \cap C^{1, \alpha}(\bar{\Omega})$ be the weak solution of (4.20).

Then for $K \geq \varrho,\left\|w_{K}\right\|_{L^{\infty}(\Omega)} \leq C_{1} K^{1 /\left(p^{-}-1\right)}, w_{K}(x) \geq C_{2} K^{1 /\left(p_{+}-1+\varsigma\right)} \operatorname{dist}(x, \partial \Omega)$ where $\varsigma \in(0,1)$ and for $K<\varrho$, $\left\|w_{K}\right\|_{L^{\infty}(\Omega)} \leq C_{3} K^{1 /\left(p^{+}-1\right)}$ where $C_{1}, C_{2}$ and $C_{3}$ depends upon $p_{+}, p_{-}, N, \Omega$. Moreover if $K_{1}<K_{2}$ then $w_{K_{1}} \leq w_{K_{2}}$.

Next we prove a slight extension of Proposition A.2 in [12].

Proposition A.1. Let $p \in C(\bar{\Omega})$ and $q \in\left(1, p_{-}\right]$. Assume $u \in \mathbf{W}$ satisfying for any $\Psi \in \mathbf{W}$ :

$$
\int_{\Omega}|\nabla u|^{p(x)-2} \nabla u . \nabla \Psi d x=\int_{\Omega} h u^{q-1} \Psi d x
$$

where $h \in L^{2}(\Omega) \cap L^{r}(\Omega)$ with $r>\max \left\{1, \frac{N}{p_{-}}\right\}$. Then $u \in L^{\infty}(\Omega)$. 
First we prove a regularity lemma.

Lemma A.1. Let $u \in W_{0}^{1, p}(\Omega)$ satisfying for any $B_{R}, R<R_{0}$, and for all $\sigma \in(0,1)$, and any $k \geq k_{0}>0$

$\int_{A_{k, \sigma R}}|\nabla u|^{p} d x \leq C\left[\int\left|\frac{u-k}{R(1-\sigma)}\right|^{p^{*}} d x+k^{\alpha}\left|A_{k, R}\right|+\left|A_{k, R}\right|^{\frac{p}{p^{*}}+\varepsilon}+k^{\beta}\left|A_{k, R}\right|^{\frac{p}{p^{*}}+\varepsilon}+\left(\int\left|\frac{u-k}{R(1-\sigma)}\right|^{p^{*}} d x\right)^{\frac{p}{p^{*}}}\left|A_{k, R}\right|^{\delta}\right]$

where $A_{k, R}=\left\{x \in B_{R} \cap \Omega \mid u(x)>k\right\}, 0<\alpha<p^{\star}=\frac{N p}{N-p}, \beta \in(1, p]$ and $\varepsilon, \delta>0$. Then $u \in L^{\infty}(\Omega)$.

Proof. A similar result exists in [23] or in [17] without the term $k^{\beta}\left|A_{k, R}\right|^{\frac{p}{p^{*}}+\varepsilon}$. For the reader's convenience, we include the complete proof.

Let $x_{0} \in \bar{\Omega}, B_{R}$ the ball centred in $x_{0}$. We define $K_{R} \stackrel{\text { def }}{=} B_{R} \cap \Omega$ and we set

$$
r_{j}=\frac{R}{2}+\frac{R}{2^{j+1}}, \quad \tilde{r}_{j}=\frac{r_{j}+r_{j+1}}{2} \text { and } k_{j}=k\left(1-\frac{1}{2^{j+1}}\right) \text { for any } j \in \mathbb{N} .
$$

Define also

$$
I_{j}=\int_{A_{k_{j}, r_{j}}}\left|u(x)-k_{j}\right|^{p^{*}} d x \text { and } \varphi(t)= \begin{cases}1 & \text { if } 0 \leq t \leq \frac{1}{2} \\ 0 & \text { if } t \geq \frac{3}{4}\end{cases}
$$

satisfying $\varphi \in C^{1}([0,+\infty))$ and $0 \leq \varphi \leq 1$. We set $\varphi_{j}(x)=\varphi\left(\frac{2^{j+1}}{R}\left(|x|-\frac{R}{2}\right)\right)$. Hence $\varphi_{j}=1$ on $B_{r_{j+1}}$ and $\varphi_{j}=0$ on $\mathbb{R}^{N} \backslash B_{\tilde{r}_{j+1}}$.

We have

$$
\begin{aligned}
I_{j+1} & =\int_{A_{k_{j+1}, r_{j+1}}}\left|u(x)-k_{j+1}\right|^{p^{*}} d x=\int_{A_{k_{j+1}, r_{j+1}}}\left|u(x)-k_{j+1}\right|^{p^{*}} \varphi_{j}(x)^{p^{*}} d x \\
& \left.\leq \int_{K_{R}}\left(u(x)-k_{j+1}\right)^{+} \varphi_{j}(x)\right)^{p^{*}} d x .
\end{aligned}
$$

Since $u \in W_{0}^{1, p}(\Omega),\left(u-k_{j+1}\right)^{+} \varphi_{j} \in W_{0}^{1, p}\left(K_{R}\right)$,

$$
\begin{aligned}
I_{j+1} & \lesssim\left(\int_{K_{R}}\left|\nabla\left(\left(u-k_{j+1}\right)^{+} \varphi_{j}\right)\right|^{p} d x\right)^{\frac{p^{\star}}{p}} \\
& \lesssim\left(\int_{A_{k_{j+1}, \tilde{r}_{j}}}|\nabla u|^{p} d x+\int_{A_{k_{j+1}, \tilde{r}_{j}}}\left(u-k_{j+1}\right)^{p} d x\right)^{\frac{p^{\star}}{p}}
\end{aligned}
$$

where we use the notation $f \lesssim g$ in the sense there exists a constant $c>0$ such that $f \leq c g$. Since $\tilde{r}_{j}<r_{j}$, we have

$$
\begin{aligned}
I_{j+1} \lesssim & \left(2^{j p^{\star}} \int_{A_{k_{j+1}, r_{j}}}\left|u-k_{j+1}\right|^{p^{\star}} d x+k_{j+1}^{\alpha}\left|A_{k_{j+1}, r_{j}}\right|+\left|A_{k_{j+1}, r_{j}}\right|^{\frac{p}{p^{*}}+\varepsilon}\right. \\
& \left.+\left.k_{j+1}^{\beta}\left|A_{k_{j+1}, r_{j}} \frac{p^{\frac{p}{p^{*}}+\varepsilon}}{2^{j p}}\left(\int_{A_{k_{j+1}, r_{j}}}\left|u-k_{j+1}\right|^{p^{*}} d x\right)^{\frac{p}{p^{*}}}\right| A_{k_{j+1}, r_{j}}\right|^{\delta}+\iint_{A_{k_{j+1}, r_{j}}}\left|u-k_{j+1}\right|^{p^{\star}} d x\right)^{\frac{p^{*}}{p}} .
\end{aligned}
$$


Moreover, for any $j, k_{j} \leq k_{j+1}$, this implies

$$
I_{j} \geq \int_{A_{k_{j+1}, r_{j}}}\left|u-k_{j}\right|^{p^{*}} d x \geq \int_{A_{k_{j+1}, r_{j}}}\left|k_{j}-k_{j+1}\right|^{p^{*}} d x=\left|A_{k_{j+1}, r_{j}} \| k_{j+1}-k_{j}\right|^{p^{*}} .
$$

Then, for any $k>k_{0}$ and $j \in \mathbb{N}$

$$
\left|A_{k_{j+1}, r_{j}}\right|+k_{j+1}^{p^{*}}\left|A_{k_{j+1}, r_{j}}\right| \lesssim 2^{j p^{*}} I_{j}
$$

where the constant in the notation depends only on $k_{0}, p$ and $\alpha$. From the previous inequality, we deduce

$$
k_{j+1}^{\beta}\left|A_{k_{j+1}, r_{j}}\right|^{\frac{p}{p^{*}}+\varepsilon} \leq k_{j+1}^{p+\varepsilon p^{*}}\left|A_{k_{j+1}, r_{j}}\right|^{\frac{p}{p^{*}}+\varepsilon} \lesssim 2^{j\left(p+\varepsilon p^{*}\right)} I_{j}^{\frac{p}{p^{x^{*}}}+\varepsilon} .
$$

Replacing in (A.5), we obtain

$$
I_{j+1} \lesssim\left(2^{j p^{*}} I_{j}+2^{j\left(p+\varepsilon p^{*}\right)} I_{j}^{\frac{p}{p^{*}}+\varepsilon}+2^{j\left(p+\delta p^{*}\right)} I_{j}^{\frac{p}{p^{*}} \delta}\right)^{\frac{p^{*}}{p}}
$$

Setting $M=\frac{p}{p^{*}} \max \left\{p^{\star}, p+\varepsilon p^{\star}, p+\delta p^{\star}\right\}$ and $\theta=\min \left\{1-\frac{p}{p^{*}}, \varepsilon, \delta\right\}$ and noting

$$
I_{j} \leq \int_{K_{R}}\left(\left|u-k_{j}\right|^{+}\right)^{p^{*}} d x \leq \int_{K_{R}}|u|^{p^{\star}} \leq\|u\|_{W_{0}^{1, p}}^{p^{\star}},
$$

(A.6) becomes

$$
I_{j+1} \lesssim 2^{j M} I_{j}^{1+\frac{\theta p^{*}}{p}}
$$

where the constant depends on $\|u\|_{W_{0}^{1, p}}, k_{0}, \alpha$ and $p$. We conclude with Lemma 4.7 in Chapter 2 of [24].

For this it suffices to prove that $I_{0}$ is small enough. Indeed $u \in L^{p^{*}}(\Omega)$ implies

$$
I_{0}=\int_{A_{\frac{k}{2}, R}}\left|u-\frac{k}{2}\right|^{p^{*}} d x \rightarrow 0 \quad \text { as } \quad k \rightarrow \infty .
$$

Hence for $k$ large enough, $I_{0} \leq C^{-\frac{1}{\eta}}\left(2^{M}\right)^{-\frac{1}{\eta^{2}}}$ with $\eta=\frac{\theta p^{*}}{p}$. Thus $I_{j}$ converges to 0 as $j \rightarrow+\infty$ and

$$
\int_{A_{k, \frac{R}{2}}}|u-k|^{p^{\star}} d x=0
$$

We deduce that $u \leq k$ on $K_{\frac{R}{2}}$. In the same way, we prove that $-u \leq k$ on $K_{\frac{R}{2}}$.

Since $\bar{\Omega}$ is compact, we conclude that $u \in L^{\infty}(\Omega)$.

Proof of Proposition A.1: We follow the idea of the proof of Theorem 4.1 in [11]. Let $x_{0} \in \bar{\Omega}, B_{R}$ the ball of radius $R$ centered in $x_{0}$ and $K_{R} \stackrel{\text { def }}{=} \Omega \cap B_{R}$. We define

$$
p^{+} \stackrel{\text { def }}{=} \max _{K_{R}} p(x) \text { and } \quad p^{-\stackrel{\text { def }}{=}} \min _{K_{R}} p(x)
$$

and we choose $R$ small enough such that $p^{+}<\left(p^{-}\right)^{\star}$ where

$$
\left(p^{-}\right)^{\star} \stackrel{\text { def }}{=} \begin{cases}\frac{N p^{-}}{N-p^{-}} & \text {if } p^{-}<N, \\ p^{+}+1 & \text { if } p^{-} \geq N .\end{cases}
$$

Fix $(s, t) \in\left(\mathbb{R}_{+}^{\star}\right)^{2}, t<s<R$ then $K_{t} \subset K_{s} \subset K_{R}$. Define $\varphi \in C^{\infty}(\Omega), 0 \leq \varphi \leq 1$ such that

$$
\varphi= \begin{cases}1 & \text { in } B_{t} \\ 0 & \text { in } \mathbb{R}^{N} \backslash B_{S}\end{cases}
$$


satisfying $|\nabla \varphi| \lesssim 1 /(s-t)$. Let $k \geq 1$, using the same notations as previously $A_{k, \lambda}=\left\{y \in K_{\lambda} \mid u(y)>k\right\}$ and taking $\Psi=\varphi^{p^{+}}(u-k)^{+} \in W_{0}^{1, p(x)}(\Omega)$ in (A.4), we obtain

$$
\int_{A_{k, s}}|\nabla u|^{p(x)} \varphi^{p^{+}} d x+p^{+} \int_{A_{k, s}}|\nabla u|^{p(x)-2} \nabla u \cdot \nabla \varphi \varphi^{p^{+}-1}(u-k)^{+} d x=\int_{A_{k, s}} h u^{q-1} \varphi^{p^{+}}(u-k) d x .
$$

Hence by Young inequality, for $\epsilon>0$, we have

$$
p^{+} \int_{A_{k, s}}|\nabla u|^{p(x)-2} \nabla u \cdot \nabla \varphi \varphi^{p^{+}-1}(u-k) d x \leq \varepsilon \int_{A_{k, s}}|\nabla u|^{p(x)} \varphi^{\left(p^{+}-1\right) \frac{p(x)}{p(x)-1}} d x+c \varepsilon^{-1} \int_{A_{k, s}}(u-k)^{p(x)}|\nabla \varphi|^{p(x)} d x .
$$

Since $|\nabla \varphi| \leq c /(s-t)$ and for any $x \in K_{R}, p^{+} \leq\left(p^{+}-1\right) \frac{p(x)}{p(x)-1}$, we have $\varphi^{\left(p^{+}-1\right) \frac{p(x)}{p(x)-1}} \leq \varphi^{p^{+}}$. This implies

$$
p^{+} \int_{A_{k, s}}|\nabla u|^{p(x)-2} \nabla u . \nabla \varphi \varphi^{p^{+}-1}(u-k) d x \leq \varepsilon \int_{A_{k, s}}|\nabla u|^{p(x)} \varphi^{p^{+}} d x+c \varepsilon^{-1} \int_{A_{k, s}}\left(\frac{u-k}{s-t}\right)^{p(x)} d x .
$$

Using Hölder inequality, we estimate the right-hand side of (A.7) as follows:

$$
\int_{A_{k, s}} h u^{q-1} \varphi^{p^{+}}(u-k) d x \leq\|h\|_{L^{r}}\left(\int_{A_{k, s}} u^{\frac{r(q-1)}{r-1}}(u-k)^{\frac{r}{r-1}} d x\right)^{\frac{r-1}{r}} .
$$

Since $r>\frac{N}{p^{-}}$, we have $\frac{\left(p^{-}\right)^{\star}}{p^{-}} \frac{r-1}{r}>1$, applying once again the Hölder inequality and the Young inequality, we obtain

$$
\int_{A_{k, s}} h u^{q-1} \varphi^{p^{+}}(u-k) d x \lesssim\left(\int_{A_{k, s}} u^{\frac{q\left(p^{-}\right)^{*}}{p^{-}}} d x+\int_{A_{k, s}}(u-k)^{\frac{q\left(p^{-}\right)^{*}}{p^{-}}} d x\right)^{\frac{p^{-}}{\left(p^{-}\right)^{*}}}\left|A_{k, s}\right|^{\delta}
$$

where $\delta=\frac{r-1}{r}-\frac{p^{-}}{\left(p^{-}\right)^{*}}>0$.

Set $A_{k, s, t}=\left\{x \in A_{k, s} \mid u(x)-k>s-t\right\}$ and its complement as $A_{k, s, t}^{c}$. Now we split the integrals in the right-hand side of (A.9) as follows:

$$
\begin{aligned}
\int_{A_{k, s, t}}\left(\frac{u-k}{s-t}\right)^{\frac{q\left(p^{-}\right)^{*}}{p^{-}}}(s-t)^{\frac{q\left(p^{-}\right)^{*}}{p^{-}}} d x & +\int_{A_{k, s, t}^{c}}\left(\frac{u-k}{s-t}\right)^{\frac{q\left(p^{-}\right)^{*}}{p^{-}}}(s-t)^{\frac{q\left(p^{-}\right)^{*}}{p^{-}}} d x \\
& \lesssim \int_{A_{k, s}}\left(\frac{u-k}{s-t}\right)^{\left(p^{-}\right)^{*}} d x+\left|A_{k, s}\right|^{\text {def }} \stackrel{\mathcal{J}}{ }
\end{aligned}
$$

since $q<p$ - and we also have

$$
\int_{A_{k, s}} u^{\frac{q\left(p^{-}\right)^{*}}{p^{-}}} d x \lesssim \int_{A_{k, s}}(u-k)^{\frac{q\left(p^{-}\right)^{*}}{p^{-}}}+k^{\frac{q\left(p^{-}\right)^{*}}{p^{-}}} d x \lesssim \mathcal{J}+k^{\frac{q\left(p^{-}\right)^{*}}{p^{-}}}\left|A_{k, s}\right| .
$$

In the same way, the second term in the right-hand side of (A.8) can be estimated as follows:

$$
\int_{A_{k, s} \cap A_{k, s, t}}\left(\frac{u-k}{s-t}\right)^{p(x)} d x+\int_{A_{k, s} \cap A_{k, s, t}^{c}}\left(\frac{u-k}{s-t}\right)^{p(x)} d x \lesssim \mathcal{J} .
$$

Finally plugging (A.8)-(A.10), we obtain for $\varepsilon$ small enough

$$
\int_{A_{k, s}}|\nabla u|^{p(x)} \varphi^{p^{+}} d x \lesssim \mathcal{J}+\left|A_{k, S}\right|^{\delta}\left(\mathcal{J}+k^{\frac{q\left(p^{-}\right)^{*}}{p^{-}}}\left|A_{k, s}\right|\right)^{\frac{p^{-}}{\left(p^{-}\right)^{*}}}
$$


where the constant depends on $p, R$ and $\varepsilon$. Moreover we have

$$
\begin{aligned}
\left(\mathcal{J}+k^{\frac{q\left(p^{-}\right)^{*}}{p^{-}}}\left|A_{k, s}\right|\right)^{\frac{p^{-}}{\left(p^{-}\right)^{*}}} & \lesssim\left(\int_{A_{k, S}}\left(\frac{u-k}{s-t}\right)^{\left(p^{-}\right)^{*}} d x\right)^{\frac{p^{-}}{\left(p^{-}\right)^{*}}} \\
& +\left|A_{k, S}\right|^{\frac{p^{-}}{\left(p^{-}\right)^{*}}}+k^{q} \mid A_{k, s} \frac{p^{-}}{\left(p^{-}\right)^{*}} .
\end{aligned}
$$

To conclude, using the Young inequality, we obtain the following estimate:

$$
\begin{gathered}
\int_{A_{k, t}}|\nabla u|^{p^{-}} d x \leq \int_{A_{k, s}}|\nabla u|^{p(x)} \varphi^{p^{+}} d x \lesssim \int_{A_{k, s}}\left(\frac{u-k}{s-t}\right)^{\left(p^{-}\right)^{*}} d x+2\left|A_{k, s}\right| \\
+\left(1+k^{q}\right)\left|A_{k, s}\right|^{\frac{p^{-}}{\left(p^{-}\right)^{*}}+\delta}+\left|A_{k, s}\right|^{\delta}\left(\int_{A_{k, s}}\left(\frac{u-k}{s-t}\right)^{\left(p^{-}\right)^{*}} d x\right)^{\frac{p^{-}}{\left(p^{-}\right)^{*}}} \cdot
\end{gathered}
$$

By Lemma A.1, we deduce that $u$ bounded in $\Omega$.

Combining Theorem 4.1 of [11] and Proposition A.1, we have the following corollary:

Corollary A.1. Let $p \in C(\bar{\Omega})$ and $q \in\left(1, p_{-}\right]$. Assume $u \in \mathbf{W}$ and nonnegative satisfying for any $\Psi \in \mathbf{W}, \Psi \geq 0$,

$$
\int_{\Omega} u^{2 q-1} \Psi d x+\int_{\Omega}|\nabla u|^{p(x)-2} \nabla u \cdot \nabla \Psi d x \leq \int_{\Omega}\left(f(x, u)+h u^{q-1}\right) \Psi d x
$$

where $f$ verifies for any $(x, t) \in \Omega \times \mathbb{R}^{+},|f(x, t)| \leq c_{1}+c_{2}|t|^{s(x)-1}$ with $s \in C(\bar{\Omega})$ such that for any $x \in \bar{\Omega}$, $1<s(x)<p^{\star}(x)$ and $h \in L^{2}(\Omega) \cap L^{r}(\Omega)$ with $r>\max \left\{1, \frac{N}{p_{-}}\right\}$. Then $u \in L^{\infty}(\Omega)$.

\section{References}

[1] M. Picone, Sui valori eccezionali di un parametro da cui dipende un'equazione differenziale lineare ordinaria del second'ordine, Ann. Scuola Norm. Sup. Pisa Cl. Sci. 11, (1910), 1-144.

[2] M. Picone, Un teorema sulle soluzioni delle equazioni lineari ellittiche autoaggiunte alle derivate parziali del secondoordine, Atti Accad. Naz. Lincei Rend. Cl. Sci. Fis. Mat. Natur. 53, (1972), 516-519.

[3] W. Allegretto and Y. Huang, A Picone's identity for the p-Laplacian and applications, Nonlinear Anal. 32, (1998), no. 7, 819-830.

[4] J. Giacomoni and P. Takáč, A p (x)-Laplacian extension of the Diaz-Saa inequality and some applications, to appear in Proceedings of Roy. Soc. Edinburgh serie A.

[5] M. Ruzicka, Electrorheological fluids: modeling and mathematical theory, Lecture Notes in Mathematics, 1748, SpringerVerlag, Berlin, 2000.

[6] Y. Chen, S. Levine and M. Rao, Variable exponent, linear growth functionals in image restoration, SIAM J. Appl. Math. 66, (2006), no. 4, 1383-1406.

[7] G. Fragnelli, Positive periodic solutions for a system of anisotropic parabolic equations, J. Math. Anal. Appl. 367, (2010), no. 1, 204-228.

[8] B. Bougherara and J. Giacomoni, Existence of mild solutions for a singular parabolic equation and stabilization, Adv. Nonlinear Anal. 4, (2015), no. 2, 123-134.

[9] L. Diening, P. Harjulehto, P. Hasto and M. Ruzicka, Lebesgue and Sobolev Spaces with Variable Exponents, Lecture Notes in Mathematics, 2017, Springer, Heidelberg, 2011.

[10] X. Fan, Global $C^{1, \alpha}$ regularity for variable exponent elliptic equations in divergence form, J. Differential Equations 235, (2007), no. 2, 397-415

[11] X. Fan and D. Zhao, A class of De Giorgi type and Hölder continuity, Nonlinear Anal. 36, (1999), no. 3, 295-318

[12] J. Giacomoni, V. Rădulescu and G. Warnault, Quasilinear parabolic problem with variable exponent: qualitative analysis and stablization, Commun. Contemp. Math. 20, (2018), no. 8.

[13] V. Rădulescu, Nonlinear elliptic equations with variable exponent: old and new, Nonlinear Anal. 121, (2015), 336-369. 
[14] V. Rădulescu and D. Repovš, Partial differential equations with variable exponents, Variational Methods and Qualitative Analysis, Monographs and Research Notes in Mathematics, CRC Press, Taylor \& Francis Group, 2015.

[15] G. Bognar and O. Dosly, The application of Picone-type identity for some non-linear elliptic differential equations, Acta Math. Univ. Comenian. 72, (2003), no. 1, 45-57.

[16] X. Cui and T. Feng, Anisotropic Picone identities and anisotropic Hardy inequalities, J. Inequal. Appl., (2017), no. 16.

[17] J. Giacomoni, S. Tiwari and G. Warnault, Quasilinear parabolic problem with $\mathrm{p}(\mathrm{x})$-Laplacian: existence, uniqueness of weak solutions and stabilization, NoDEA Nonlinear Differential Equations Appl. 23, (2016), no. 3, Art. 24.

[18] H. Brezis, Functional Analysis, Sobolev Spaces and Partial Differential Equations, Springer, New York, 2011.

[19] Q. Zhang, A strong maximum principle for differential equations with nonstandard $p(x)$-growth conditions, J. Math. Anal. Appl. 312, (2005), no. 1, 24-32.

[20] V. Barbu, Nonlinear differential equations of monotone types in Banach spaces, Springer Monographs in Mathematics, Springer, New York, 2010.

[21] P. Takáč, L. Tello and M. Ulm, Variational Problems with a p-homogeneous energy, Positivity 6, (2001), no. 1, 75-94.

[22] X. Fan, On the sub-supersolution method for p(x)-Laplacian equations, J. Math. Anal. Appl. 330, (2007), no. 1, 665-682.

[23] N. Fusco and C. Sbordone, Some remarks on the regularity of minima of anisotropic integrals,Comm. Partial Differential Equations 18, (1993), no. 1-2 153-167.

[24] O. A. Ladyzhenskaya and N. N. Ural'tseva, Linear and quasilinear elliptic equations, Leon Ehrenpreis Academic Press, New York-London, 1968. 